\title{
Article \\ A Genomic and Transcriptomic Study on the DDT-Resistant Trichoderma hamatum FBL 587: First Genetic Data into Mycoremediation Strategies for DDT-Polluted Sites
}

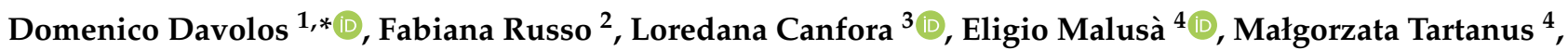

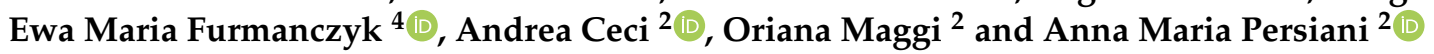 \\ 1 Department of Technological Innovations and Safety of Plants, Products and Anthropic Settlements (DIT), \\ INAIL, Research Area, Via R. Ferruzzi 38/40, 00143 Rome, Italy \\ 2 Department of Environmental Biology, Sapienza University of Rome, P.le A. Moro 5, 00185 Rome, Italy; \\ fabiana.russo1784@gmail.com (F.R.); andrea.ceci@uniroma1.it (A.C.); oriana.maggi@gmail.com (O.M.); \\ annamaria.persiani@uniroma1.it (A.M.P.) \\ 3 Council of Agricultural Research and Economics, Centre for Agriculture and Environment, \\ Via Della Navicella 2/4, 00184 Rome, Italy; loredana.canfora@crea.gov.it \\ 4 The National Institute of Horticultural Research, ul. Konstytucji 3 Maja 1/3, 96-100 Skierniewice, Poland; \\ eligio.malusa@inhort.pl (E.M.); malgorzata.tartanus@inhort.pl (M.T.); ewa.furmanczyk@inhort.pl (E.M.F.) \\ * Correspondence: d.davolos@inail.it; Tel.: +39-0654876328
}

Citation: Davolos, D.; Russo, F.; Canfora, L.; Malusà, E.; Tartanus, M.; Furmanczyk, E.M.; Ceci, A.; Maggi, O.; Persiani, A.M. A Genomic and Transcriptomic Study on the DDT-Resistant Trichoderma hamatum FBL 587: First Genetic Data into Mycoremediation Strategies for DDT-Polluted Sites. Microorganisms 2021, 9, 1680. https://doi.org/ $10.3390 /$ microorganisms 9081680

Academic Editor: Lisa M. Gieg

Received: 3 July 2021

Accepted: 4 August 2021

Published: 7 August 2021

Publisher's Note: MDPI stays neutral with regard to jurisdictional claims in published maps and institutional affiliations.

Copyright: (c) 2021 by the authors. Licensee MDPI, Basel, Switzerland. This article is an open access article distributed under the terms and conditions of the Creative Commons Attribution (CC BY) license (https:// creativecommons.org/licenses/by/ $4.0 /)$.
Abstract: Trichoderma hamatum FBL 587 isolated from DDT-contaminated agricultural soils stands out as a remarkable strain with DDT-resistance and the ability to enhance DDT degradation process in soil. Here, whole genome sequencing and RNA-Seq studies for T. hamatum FBL 587 under exposure to DDT were performed. In the $38.9 \mathrm{Mb}$-genome of T. hamatum FBL 587, 10,944 protein-coding genes were predicted and annotated, including those of relevance to mycoremediation such as production of secondary metabolites and siderophores. The genome-scale transcriptional responses of T. hamatum FBL 587 to DDT exposure showed 1706 upregulated genes, some of which were putatively involved in the cellular translocation and degradation of DDT. With regards to DDT removal capacity, it was found upregulation of metabolizing enzymes such as P450s, and potentially of downstream DDT-transforming enzymes such as epoxide hydrolases, FAD-dependent monooxygenases, glycosyland glutathione-transferases. Based on transcriptional responses, the DDT degradation pathway could include transmembrane transporters of DDT, antioxidant enzymes for oxidative stress due to DDT exposure, as well as lipases and biosurfactants for the enhanced solubility of DDT. Our study provides the first genomic and transcriptomic data on T. hamatum FBL 587 under exposure to DDT, which are a base for a better understanding of mycoremediation strategies for DDT-polluted sites.

Keywords: bioremediation; carbohydrate active enzymes (CAZymes); DDT biodegradation; fungal genomics; genome-scale RNA-Seq; Hypocreaceae; secondary metabolites; siderophores; Trichoderma hamatum; whole genome sequencing

\section{Introduction}

Anthropogenic halogenic pollutants such as 1,1,1-Trichloro-2,2-bis (4-chlorophenyl) ethane (DDT) and its metabolite products (e.g., 1,1-dichloro-2,2-bis (4-chlorophenyl) ethane (DDD), and 1,1-dichloro-2,2-bis (4-chlorophenyl) ethylene (DDE)) have accumulated in soils and pose significant health and environmental concerns [1,2]. In particular DDT-polluted agricultural sites are of great human health concern due to the environmental persistence of DDT and its metabolite products becoming long-term sources of exposure [3].

Mycoremediation of polluted areas is a promising method to remove many hazardous pollutants [4]. The biodegradation of DDT using microfungi has been regarded as an efficient method to remove DDT in DDT-polluted sites without causing significant ecological consequences [5]. 
It has been reported that DDT-resistant microfungi can efficiently degrade DDT, but the metabolic pathways they use are not yet fully understood. Recently, species belonging to Trichoderma genus with the ability to metabolize a variety of xenobiotics including DDT and polycyclic aromatic hydrocarbons (PAHs) have been studied [6]. Trichoderma hamatum is included among PAH-degrading species, which uses multicopper laccases, ring-cleavage dioxygenases and peroxidases for their degradation [7,8]. The saprotroph Trichoderma hamatum FBL 587, collected from DDT-contaminated agricultural soils in Poland, has previously been screened by tolerance in the presence of DDT $(1 \mathrm{mg} / \mathrm{L})$ and regarded as a potential autochthonous fungal strain suitable for mycoremediation of DDT-contaminated agricultural soils [5].

It is well known that some filamentous fungi encode genes involved in the cellular translocation of xenobiotics, as well as in the efflux transporters to detoxify xenobiotics; the gene expression products allow their modification (phase I), conjugation (phase II) and secretion (phase III) [9]. Phase I metabolizing enzymes (cytochrome P450s, and monooxygenases) catalyse xenobiotics mainly through hydroxylation/oxidation reactions, phase II conjugating enzymes (e.g., glutathione S-transferase and sulfotransferase) add polar molecules onto xenobiotics, producing water-soluble metabolites, and the phase III secretion system consists of ATP-binding cassette (ABC) and other transmembrane transporters (e.g., major facilitator superfamily (MFS) transporters) that actively export xenobiotic and/or metabolized compounds across the cell membranes [10,11]. Even though the genomic characterization of several strains of Trichoderma species [12] and of few strains of T. hamatum has been performed [13-15], genomic and transcriptomic studies of tolerance and degradation of DDT have not been conducted on Trichoderma species. Therefore, the identification of DDT-responsive genes encoded in the Trichoderma genome is still lacking. In order to expand our knowledge on the genetic basis for the DDT resistance and removal capability of T. hamatum FBL 587, we sequenced and analysed its genome to provide a foundation of comprehensive understanding regarding the application of this strain for mycoremediation strategies of DDT-polluted sites.

Here we present the $38.9 \mathrm{Mb}$-genome sequence of T. hamatum FBL 587. We performed an analysis of 10,944 protein-coding genes that were predicted and annotated, focusing on those associated to carbohydrate-active enzymes (CAZymes), secondary metabolites (SMs), and siderophores, all of relevance to mycoremediation. In the genome we also investigated genetic repertoires potentially involved in the resistance and metabolism of DDT. Moreover, transcriptomic analysis was performed to observe differential expression of genes by DDT $(10 \mathrm{mg} / \mathrm{L})$. The genome-scale RNA-Seq study performed on exposure of T. hamatum FBL 587 to DDT highlights genetic features that shed a light on the DDT tolerance and removal by this strain. Finally, the DDT-degradation performance of the strain was tested in a pot experiment.

\section{Materials and Methods}

\subsection{DDT Degradation Process in Soil by T. hamatum FBL 587}

A pot experiment was performed to assess the potential additive impact of Trichoderma hamatum (Bonord.) Bainier FBL 587 application on DDT phytoremediation capacity of two different Cucurbita pepo accessions, a species with well described and documented potential in bioremediation [16]. Soil originating from a field that in several preliminary analyses did not show any contamination with DDT was spiked in with analytical grade $\mathrm{p}, \mathrm{p}^{\prime}$-DDT (Sigma Aldrich). A stock solution of DDT in acetone was mixed with water $(3: 97 v / v)$ to prepare the working solution applied to the soils. The DDT solution was uniformly sprayed using a hand sprayer onto $20 \mathrm{~L}$ of homogenised soil arranged as a 2 -cm-thick layer over a plastic film, in a quantity necessary to deliver $0.125 \mathrm{mg}$ of analytical grade $\mathrm{p}, \mathrm{p}^{\prime}$-DDT per litre of soil. However, the initial concentration of DDT in soil was determined. The contamination procedure was performed individually for the soil used for growing each selected accession for the bioremediation experiments. Two C. pepo accessions were selected for this study: AMES 26607 and PI 614701 grown from seeds 
obtained from USDA Germplasm Repository. The seeds were germinated in Petri dishes under controlled conditions $\left(25^{\circ} \mathrm{C}\right.$ and $\left.80 \% \mathrm{RH}\right)$ and after about two weeks, on 27 July 2019 they were planted in artificially DDT-contaminated soil not sterilized. On 30 August the plants were watered with $400 \mathrm{~mL} /$ pot of $T$. hamatum FBL $587\left(4 \times 10^{4}\right.$ spores $\left./ \mathrm{mL}\right)$, precultured on Malt extract agar (MEA) at $25{ }^{\circ} \mathrm{C}$ in the dark. Plants treated with water were used as control. The experiment, done in triplicate ( 3 pots each having 2 plants), was ended on 9 October. The plants were separated from soil and each plant fraction (root, aboveground and sometimes fruits) together with remaining soil were analysed for DDT residues. DDT and its metabolites were determined as described elsewhere [3].

\subsection{Genomic DNA Extraction, Whole Genome Sequencing and Bioinformatics Analyses}

Genomic DNA was extracted from mycelial samples of T. hamatum FBL 587 using the QIAamp DNA Microbiome Kit (Qiagen, Hilden, Germany) and was used for Illumina library preparation. DNA quality and concentration were assessed with Nanodrop 2000 (Thermo Fisher Scientific, Wilmington, DE, USA). The paired-end genomic libraries $(2 \times 250 \mathrm{bp})$ were built using the MiSeq v.3 reagents (600 cycles) and sequenced at Eurofins Genoma Group (Rome, Italy).

Reads quality was assessed with fastQC v.0.11.9 [17], and adapters removal was performed with Trimmomatic v.0.38 [18]. The filtered reads were de novo assembled using SPAdes v. 3.11 [19] with default parameters, in combination with BayesHammer (distributed with SPAdes latest version) [20] for reads error correction. Nuclear genome statistics were calculated using QUAST v.4.5 [21]. Ribosomal RNA (rRNA) and transferRNA (tRNA) were predicted with RNAmmer v.1.2 [22] and tRNAscan-SE v. 2.0 [23], respectively. Simple repeats and low complexity DNA sequences were detected by the program RepeatMasker with the search engine HMMER on Trichoderma [24].

The quality assessment for genome assembly, the gene structure annotations, and the completeness of the assembly genome was assessed using GenomeQC [25]. De novo gene prediction was performed with Augustus v.2.5.5 [26] using Fusarium graminearum as the training species. Functional annotation was performed with PANNZER2 [27]. Protein function prediction assigned Gene Ontology (GO) terms to proteins, specifying molecular functions, involvement in biological processes and subcellular localizations. For the enzymes obtained from sequenced genomes, protein IDs (ProtID) are used as gene identifiers, while for other enzymes, GenBank accession number (GbID) are provided.

Carbohydrate-Active enZYmes (CAZymes; http:/ / www.cazy.org, accessed on 1 June 2021) for complex carbohydrate metabolism were identified with the dbCAN2 [28], which integrates three state-of-the-art tools for CAZome (all CAZymes of a genome) annotation: (i) HMMER search (E-Value $<1 \times 10^{-15}$, coverage $>0.35$ ) against the dbCAN HMM (Hidden Markov Model) database; (ii) DIAMOND search $\left(E\right.$-Value $<1 \times 10^{-102}$ ) against the CAZy pre-annotated CAZyme sequence database and (iii) Hotpep search (frequency $>2.6$, hits > 6) against the conserved CAZyme short peptide database.

Secondary metabolites (SMs) biosynthesis gene clusters (BGCs) identification and characterization were performed with antiSMASH v. 6.0.0 [29]. AntiSMASH detects genome regions containing biosynthetic gene clusters based on conserved biosynthetic enzymes from different biosynthetic pathways. The antiSMASH database (available at https:/ / antismashdb.secondarymetabolites.org/, accessed on 1 June 2021) contains 177 fungal representative high-quality genomes. The identified regions were compared against a dataset of manually curated biosynthetic gene clusters with known products from the MIBiG reference database. Four Trichoderma genomes of the section Trichoderma (see [15]) and the genome of T. lixii MUT3171 [4] of the clade Harzianum/Virens (see [15]) are included in the antiSMASH analysis. Trichoderma atroviride IMI 206040 (ATCC 20476) is the representative Trichoderma high-quality genome in the antiSMASH database (https: / / antismashdb.secondarymetabolites.org, accessed on 1 June 2021; [30]), and T. lixii MUT3171 was chosen to represent the outgroup species. 


\subsection{RNA Extraction and $m R N A$ Sequencing}

Five millilitres of spore suspension $\left(5 \times 10^{6}\right.$ conidia/mL) from T. hamatum FBL 587 were added to $250 \mathrm{~mL}$ flask with sterilized $95 \mathrm{~mL}$ of Potato Dextrose Broth (PDB) and incubated for $48 \mathrm{~h}$ at $25^{\circ} \mathrm{C}$ in the dark on a rotary shaker (150 rpm, ASAL 711/D). Then, DDT was added to each $250 \mathrm{~mL}$ flask to get the final concentration of $10 \mathrm{mg} / \mathrm{L}$, except in the control. After the DDT addition, both control and treatment, prepared in triplicate, were incubated for $48 \mathrm{~h}$ at $25^{\circ} \mathrm{C}$ in the dark.

The RNA sequencing experiment was performed on six samples. RNA-Seq studies were performed on three replicates for both control and treatment (exposure to DDT at $10 \mathrm{mg} / \mathrm{L}$ for $48 \mathrm{~h}$ ). For transcriptomic analysis, total RNA was extracted from fresh $100 \mathrm{mg}$ of fungal biomass from 2 days of culture. The grown mycelia prepared in triplicate were harvested from the culture media by centrifugation and washed twice with $50 \mathrm{~mL}$ distilled water. The mycelium pellet was immediately homogenized using a Powerlyzer ${ }^{\circledR}$ 24 homogenizer (QIAGEN, Germantown, MD, USA) and total RNA was isolated using TRI Reagent (Sigma-Aldrich, St. Louis, MO, USA). Briefly, $1 \mathrm{~mL}$ of TRI Reagent was added to $100 \mathrm{mg}$ of T. hamatum FBL 587 in RNase-free and DNase-free microtubes and shaken vigorously for $45 \mathrm{~s}$ in Powerlyzer ${ }^{\circledR} 24$ homogenizer, (QIAGEN). After homogenizing the samples with TRI Reagent, the mixture was centrifuged at $12,000 \times g$ for $10 \mathrm{~min}$ at $4{ }^{\circ} \mathrm{C}$ to remove the insoluble material. The supernatant containing RNA and proteins was transferred to a new microtube, in which cold absolute chloroform $(0.2 \mathrm{~mL}$ per $\mathrm{mL}$ of TRI Reagent used) was added. The homogenate was incubated at room temperature for $15 \mathrm{~min}$ and then centrifuged at $12,000 \times g$ for $15 \mathrm{~min}$ at $4{ }^{\circ} \mathrm{C}$ to separate into a clear upper aqueous layer (containing RNA), an interphase, and a red lower organic layer (containing the DNA and proteins). Total RNA was precipitated from the aqueous layer with $1 \mathrm{~mL}$ of absolute 2-propanol (>99.5\%, molecular grade; Sigma-Aldrich) per mL of TRI Reagent used for $10 \mathrm{~min}$ at room temperature. The mixture was centrifuged at $12,000 \times \mathrm{g}$ for $10 \mathrm{~min}$ at $4{ }^{\circ} \mathrm{C}$ to allow the precipitation of the RNA pellets. The precipitated RNA was washed twice with $1 \mathrm{~mL}$ of $75 \%$ ethanol to remove impurities, vortexed and centrifuged at $7500 \times g$, at $4{ }^{\circ} \mathrm{C}$ for $5 \mathrm{~min}$, and then re-suspended in DEPC-treated MiniQuantum (deionised) water and stored at $-80{ }^{\circ} \mathrm{C}$ for further use.

Purity and quality of RNA was determined at Genomix4Life (Salerno, Italy) via NanoDrop spectrophotometer and Agilent Bioanalyzer 2100 (Agilent Technologies, Inc., Santa Clara, CA, USA), and a minimal RNA integrity number (RIN) of 7.0 was required to continue with the analysis. An RNA library was constructed using TruSeq Stranded mRNA kit (IL, San Diego, CA, USA) according to manufacturer's instructions. The sequencing of the RNA library was carried out in NextSeq 500 Illumina system using a single-end approach $(1 \times 75 \mathrm{bp})$ producing $27.5 \mathrm{M}$ of reads per sample, on average (Supplementary Table S1).

\subsection{Transcriptome Profiling with RNA-Seq Approach}

A quality check was performed on the raw sequencing data, removing low quality portions while preserving the longest high quality part of NGS reads (Supplementary Table S1); the minimum length was set to $35 \mathrm{bp}$ and the quality score to 25 by means of the software BBDuk (https:/ /jgi.doe.gov/data-and-tools / bbtools/, accessed on 1 June 2021).

The alignment of the high quality reads against the T. hamatum FBL 587 genome (present study) was performed with STAR aligner version 2.5.2b [31].

FeatureCounts version 1.5.1 [32] was used to calculate gene expression values as raw fragment counts. Normalization was applied to the raw fragment counts by using the Trimmed Mean of $M$-values (TMM; Supplementary Table S2: TMM_table.xlsx) and Reads Per Kilobase Million (RPKM; Supplementary Table S3: RPKM_table.xlsx). All the statistical analyses were performed with R (v 4.1) with the packages HTSFilter and edgeR [33].

The HTSFilter package, which implements a filtering procedure for replicated transcriptome sequencing data based on a Jaccard similarity index, was used to remove genes either not-expressed or showing high variety in the transcript expression among replicates 
(Supplementary Figure S1). The edgeR determines differential expression using empirical Bayes estimation and exact tests based on a negative binomial model.

To check if the RNA-Seq data from different conditions correlate to each other, a principal component analysis (PCA) was performed. The analysis of the RNA-Seq read counts to each annotated locus was performed in order to identify differential expression of genes by exposure to DDT among samples, i.e., with a FDR (False Discovery Rate (Benjamini-Hochberg correction) $\leq 0.05$. Among the significantly regulated genes $(\mathrm{FDR} \leq 0.05), \log 2 \mathrm{FC}$ (fold change relative to control) $\geq 1$ indicated upregulated genes while $\log 2 \mathrm{FC} \leq 1$ indicated downregulated genes.

\subsection{Accession Numbers of the Genome and Transcriptome Sequences}

The whole genome sequence project of T. hamatum FBL 587 has been deposited at DDBJ/ENA/GenBank under the accession SEIV00000000 (BioProject PRJNA513966, BioSample SAMN10717514; Sequence Read Archive (SRA) SRS5810831). The version described in this paper is version SEIV01000000.

The RNA-sequencing data have been deposited in NCBI SRA under the following IDs: SRR14056734-5-6 for DDT-treatment samples, and SRR14056737-8-9 for control samples (BioProject PRJNA513966).

\section{Results}

\subsection{DDT Degradation Process in Soil by T. hamatum FBL 587}

The initial concentration of DDT in the artificially contaminated soil ranged between 0.148 and $0.152 \mathrm{mg} / \mathrm{kg}$. The proportion among DDT metabolites in the soil before the treatment was similar among the tested variants and the contamination was determined mainly by the p, $\mathrm{p}^{\prime}$-DDT (68-69\% of total). In all cases, a decrease in the $\Sigma$ DDT (sum of all metabolites and isomers) concentration was observed at the end of the experiment: it ranged between $57 \%$ to $84 \%$ of the initial contamination, with the most promising results for combined treatment with C. pepo PI 614701 and T. hamatum FBL 587 (Figure 1). Interestingly, the main metabolite detected in soil after the treatment, regardless of the studied variant, was $\mathrm{p}, \mathrm{p}^{\prime}$-DDE. Uptake of DDT by C. pepo was confirmed by the determination of residues in both root and above-ground parts of the plant, but without translocation to the edible parts (for detailed result in Supplementary Table S4). Soil inoculation with T. hamatum FBL 587 decreased the overall DDT concentration in soil and induced an increased accumulation of DDT in roots of both C. pepo accessions. However, soil inoculation with T. hamatum FBL 587 did not affect the capacity of the plant to translocate the DDT to the above-ground tissues.

\begin{tabular}{|c|c|c|c|c|}
\hline & $\begin{array}{c}\text { Soil } \\
\text { before treatment }\end{array}$ & $\begin{array}{c}\text { Soil } \\
\text { after treatment }\end{array}$ & $\begin{array}{l}\text { C. pepo } \\
\text { roots }\end{array}$ & $\begin{array}{c}\text { C. pepo } \\
\text { shoots and leaves }\end{array}$ \\
\hline $\begin{array}{r}\text { AMES } 26607 \\
\text { Control }\end{array}$ & $\Sigma \mathrm{DDT}=0.152 \mathrm{mg} / \mathrm{kg}$ & $\Sigma \mathrm{DDT}=0.108 \mathrm{mg} / \mathrm{kg}$ & $\Sigma \mathrm{DDT}=0.123 \mathrm{mg} / \mathrm{kg}$ & $\Sigma \mathrm{DDT}=0.006 \mathrm{mg} / \mathrm{kg}$ \\
\hline $\begin{array}{r}\text { AMES } 26607 \\
+ \text { FBL } 587\end{array}$ & $\Sigma \mathrm{DDT}=0.152 \mathrm{mg} / \mathrm{kg}$ & $\Sigma \mathrm{DDT}=0.104 \mathrm{mg} / \mathrm{kg}$ & $\Sigma \mathrm{DDT}=0.243 \mathrm{mg} / \mathrm{kg}$ & $\Sigma \mathrm{DDT}=0.013 \mathrm{mg} / \mathrm{kg}$ \\
\hline $\begin{array}{r}\text { PI } 614701 \\
\text { Control }\end{array}$ & $\Sigma \mathrm{DDT}=0.148 \mathrm{mg} / \mathrm{kg}$ & $\Sigma \mathrm{DDT}=0.124 \mathrm{mg} / \mathrm{kg}$ & $\Sigma D D T=0.365 \mathrm{mg} / \mathrm{kg}$ & $\Sigma \mathrm{DDT}=0.012 \mathrm{mg} / \mathrm{kg}$ \\
\hline $\begin{array}{l}\text { PI } 614701 \\
+ \text { FBL } 587\end{array}$ & $\Sigma \mathrm{DDT}=0.148 \mathrm{mg} / \mathrm{kg}$ & $\Sigma \mathrm{DDT}=0.085 \mathrm{mg} / \mathrm{kg}$ & $\Sigma \mathrm{DDT}=0.412 \mathrm{mg} / \mathrm{kg}$ & $\Sigma \mathrm{DDT}=0.014 \mathrm{mg} / \mathrm{kg}$ \\
\hline & $p, p^{\prime}-D D D$ & $0, p^{\prime}-D D D$ & p,p'-DDT & o,p'-DDT \\
\hline
\end{tabular}

Figure 1. Effect of the soil inoculation with T. hamatum FBL 587 on the DDT degradation in soil and phytoremediation capacity of two C. pepo accessions. The percentage of each DDT metabolite or residue detected in each fraction and treatment are represented by color bars. The total concentration of DDT metabolites and isomers ( $\Sigma$ DDT) is indicated for each treatment. 


\subsection{Genome Properties of T. hamatum FBL 587}

The draft genome sequence of FBL 587 was $38.96 \mathrm{Mbp}$ long with a GC content of $48.54 \%$. In total, 10,994 protein-coding genes were predicted in the genome, of which $8564(79 \%)$ were assigned to different GO terms. The genome contains single copies of $18 \mathrm{~S}$ and $28 \mathrm{~S}$ rRNAs, $508 \mathrm{~S}$ rRNAs, 230 tRNAs and 14 pseudogenes (Table 1). We found $1.2 \%$ repetitive elements for FBL 587, with the most contribution due to simple repeats (Table 1). As shown in Table 1, the genome was $98.5 \%$ complete as predicted by the benchmarking universal single-copy ortholog (BUSCO) in GenomeQC [25]. Among the BUSCO genes, $98.5 \%$ (including $0.6 \%$ duplicated genes) were found in the assembly, as calculated with BUSCO version 3.0.2 using the trained AUGUSTUS species: Fusarium graminearum and "Pezizomycotina_odb9" data set. More statistics on nuclear genome assembly and annotation are provided in Table 1.

Table 1. Summary of the T. hamatum FBL 587 genome sequencing and assembly results.

\begin{tabular}{|c|c|}
\hline Parameter & Estimated Value \\
\hline Number reads & $6,329,382$ \\
\hline Number used reads & $6,251,876$ \\
\hline Number of contigs ( $\geq 500 \mathrm{bp})$ & 1354 \\
\hline Largest contig & 347,610 \\
\hline N50 reads & 69,288 \\
\hline L50 contigs & 166 \\
\hline Predicted genes & 10,944 \\
\hline Genome size & $38,965,975 \mathrm{bp}$ \\
\hline GC content & $48.54 \%$ \\
\hline tRNA & 230 \\
\hline 8S rRNA & 50 \\
\hline 18S rRNA & 1 \\
\hline $28 \mathrm{~S}$ rRNA & 1 \\
\hline \multicolumn{2}{|l|}{ Repeat class/family } \\
\hline Simple repeats & $399,867(1 \%)$ \\
\hline Low complexity & $79,381(0.2 \%)$ \\
\hline Completeness & $98.5 \%$ \\
\hline Fragmented & $0.7 \%$ \\
\hline Missing & $0.2 \%$ \\
\hline Complete and duplicated copy & $0.6 \%$ \\
\hline
\end{tabular}

\subsection{Prediction of Genes Encoding for CAZymes, SMs and Siderophores}

The genomic analysis of T. hamatum FBL 587 revealed the presence of a broad range of genes encoding for CAZymes, proteins involved in the production of SMs and siderophores, all of possible relevance to mycoremediation.

A genomic repository of CAZymes allowed to analyze the pattern of carbohydrate metabolism. In T. hamatum FBL 587 genome, selecting HMMER with dbCAN domain assignment we identified a total of 445 CAZymes (302 when considering all three different tools; Figure 2), including 66 auxiliary activities (AA), 4 carbohydrate-binding modules (CBM), 20 carbohydrate esterases (CE), 249 glycoside hydrolases (GH), 94 glycosyltransferases (GT), and 11 polysaccharide lyases (PL) (a full account of all the CAZymes is presented in Supplementary Table S5). 


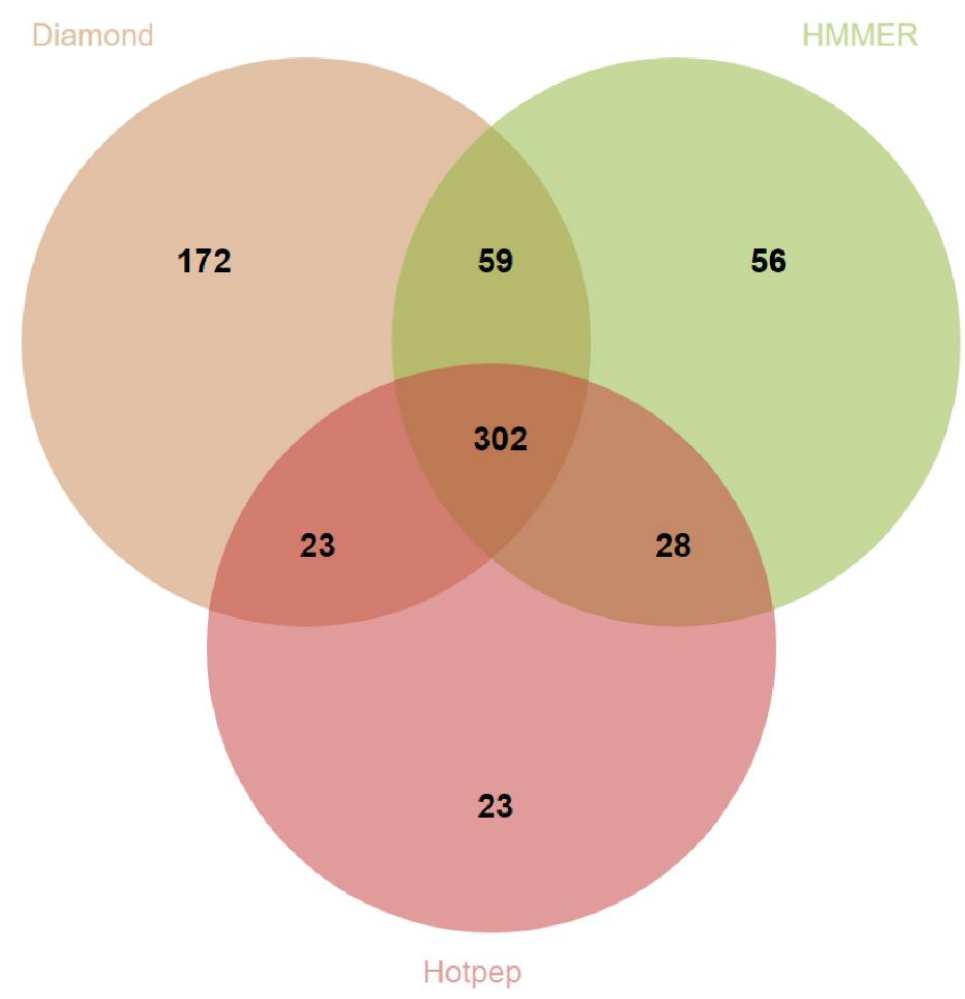

Figure 2. Venn diagram showing overlaps among the annotation results in T. hamatum FBL 587 for multi-domain CAZymes of three different tools: (1) HMMER, (2) DIAMOND, (3) Hotpep (see text).

With respect to genes involved in the formation of SMs, a class of genes of relevance to mycoremediation, in FBL 587 a genomic repository of potential SMs driven by nonribosomal peptide synthases (NRPS), polyketide synthases (PKS), hybrids NRPS-PKS and terpene was surveyed. Using the tool antiSMASH, version 6.0.0 (see [29]), we identified a relatively few number (27) of candidate BGCs for the production of SMs in T. hamatum FBL 587 (Table 2; Supplementary Table S6). Most of the antiSMASH-6.0-detected gene clusters cannot be annotated as their composition is not similar to any known fungal BGC (Supplementary Table S6). In T. hamatum FBL 587, we identified 8 PKS, 4 NRPS, 4 NRPS-PKS hybrids, and 6 terpene gene clusters (Table 2). The SM gene clusters found in the other Trichoderma species included in the paper for the antiSMASH-6.0 based-analysis are reported in Table 2 and Supplementary Tables S7-S11.

Table 2. The identification and characterization of PKS, NRPS, PKS-NRPS hybrids and terpene gene clusters in T. hamatum FBL587 and other four Trichoderma species of the section Trichoderma (see [15]) performed with antiSMASH v. 6.0.0 (see [29]). The genome of T. lixii MUT3171 [4] of the clade Harzianum/Virens (see [15]) is included in the antiSMASH-6.0 analysis, as it was chosen to represent the outgroup species. All Trichoderma genome sequences were taken from NCBI databases and have been published [4,14,15,34,35]. Genome assemblies are available at DDBJ/EMBL/GenBank under the following GenBank accessions: T. atroviride IMI 206040, ABDG02000000; T. gamsii T6085, JPDN00000000; T. asperellum CBS 433.97, MBGH00000000; T. hamatum GD12, ANCB00000000; T. lixii MUT3171, SESN00000000.

\begin{tabular}{ccccccc}
\hline Genome & NRPS, T1PKS & NRPS & NRPS-Like & T1PKS & Terpene & Total \\
\hline Trichoderma asperellum CBS 433.97 & 6 & 10 & 10 & 11 & 11 & 48 \\
\hline Trichoderma atroviride IMI 206040 & 4 & 10 & 10 & 12 & 8 & 44 \\
\hline Trichoderma gamsii T6085 & 4 & 9 & 11 & 12 & 7 & 43 \\
\hline Trichoderma hamatum GD12 & 5 & 5 & 6 & 7 & 5 & 6 \\
\hline Trichoderma hamatum FBL 587 & 4 & 4 & 5 & 7 & 27 \\
\hline Trichoderma lixii MUT3171 & 9 & 4 & 7 & 19 & 8 \\
\hline
\end{tabular}


In the genome of T.hamatum FBL 587 (node_397_length_30509_cov_30.411329),g8761 was identified using antiSMASH version 6.0.0 as the gene that shares homology with the T1PKS gene belonging to a known gene cluster, MIBiG accession BGC0001284, in Parastagonospora nodorum required for the production of alternariol (Supplementary Table S6). Alternariol is a compound that showed significant radical-scavenging (antioxidant) activity [36]. Our antiSMASH-based analysis found this putative T1PKS gene cluster for alternariol biosynthesis also in T. hamatum GD12, as reported in Supplementary Table S10.

Our antiSMASH-6.0-based analysis also demonstrated that in T. hamatum FBL 587 a BGC containing a squalene synthase (ProtID g4482) showed a composition similar (40\%) to a known cluster (MIBiG accession BGC0001839) for biosynthesis of squalestatin S1 or squalestatin analogues [37], as reported in Supplementary Table S6. This putative squalestatin S1 gene cluster was also identified in the genome of T. hamatum GD12, T. atroviride IMI 206040, T. gamsii T6085, T. asperellum CBS 433.97, and T. lixii MUT3171 (Supplementary Tables S7-S11).

Moreover, in T. hamatum FBL 587 (node_21_length_162673_cov_29.833026), g1187 encoding a member of the terpene cyclase/mutase family was identified through sequence analysis using antiSMASH v. 6.0.0 as the gene that shares homology (48\% at amino acid level) with a gene encoding for oxidosqualene cyclase (GbID ACF70484; GbID HYPSUDRAFT_48575) belonging to the antitumor clavaric acid biosynthetic gene cluster, MIBiG accession BGC0001248, of the basidiomycete Hypholoma sublateritium [38,39], as reported in (Supplementary Table S6). The antiSMASH-based analysis identified this putative gene cluster in the other Trichoderma genomes of the section Trichoderma included in this paper, namely T. hamatum GD12, T. gamsii T6085, T. asperellum CBS 433.97, and T. atroviride IMI 206040, while it was not found in the outgroup species T. lixii MUT3171, as shown in Supplementary Tables S7-S11.

With respect to genes involved in the formation of siderophores, our antiSMASH-6.0based analysis demonstrated that in T. hamatum FBL 587 a NRPS gene cluster (node_221; 29,375 nt; Supplementary Table S6) showed a composition 100\% similar to the known BGC for the production of extracellular siderophore dimethylcoprogen (MIBiG accession BGC0001249) from Alternaria alternata [40,41]. The putative dimethylcoprogen gene cluster in the genome of T. hamatum FBL 587 consisted of at least of eight genes (Figure 3), which encode for a homologue to transmembrane transporter (ProtID g7985), a homologue to ABC transmembrane transporter (ProtID g7986), a homologue to oxidoreductase (ProtID g7987), a homologue to the siderophore iron transporter (ProtID g7988), a homologue to acetyltransferase activity (ProtID g7989), a homologue to long-chain acyl-CoA synthetase (ProtID g7990), a NRPS protein (ProtID g7991), and a hypothetical protein (protein of unknown function; ProtID g7992). The putative extracellular siderophore dimethylcoprogen gene cluster was also identified in the genome of T. hamatum GD12, T. atroviride IMI 206040, T. gamsii T6085, T. asperellum CBS 433.97, and T. lixii MUT3171 (Supplementary Tables S7-S11). 


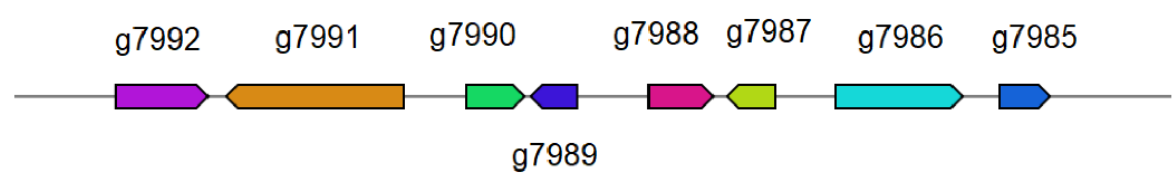

T. atroviride IMI 206040
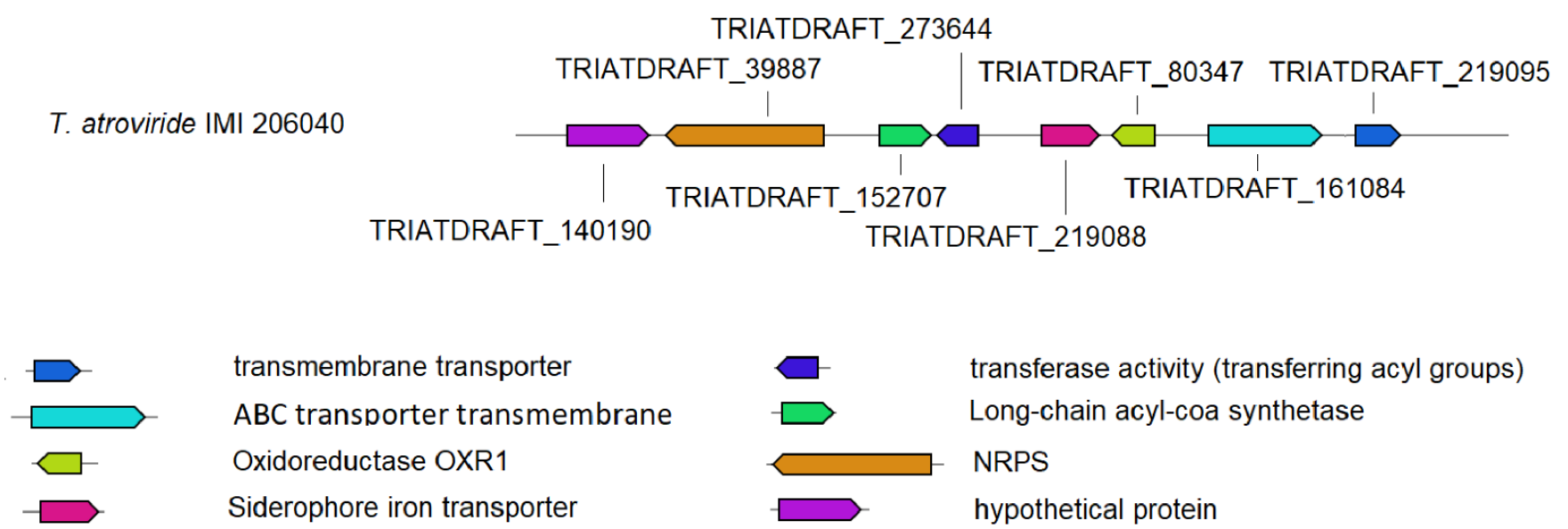

transmembrane transporter
$\mathrm{ABC}$ transporter transmembrane
Oxidoreductase OXR1
Siderophore iron transporter

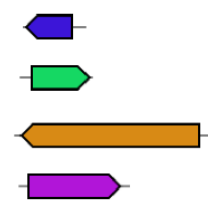

transferase activity (transferring acyl groups)
Long-chain acyl-coa synthetase
NRPS
hypothetical protein

Figure 3. The putative extracellular siderophore dimethylcoprogen gene cluster identified using the tool antiSMASH, version 6.0.0 (see [29]) in the genome of T. hamatum FBL 587. For clarity, only the orthologue gene cluster from T. atroviride IMI 206040 (representative Trichoderma high-quality genome in the antiSMASH database; https:/ / antismashdb.secondarymetabolites. org, accessed on 1 June 2021; [30]) was used for comparison. In T. hamatum FBL 587, the candidate BGC genes were assembled to a putative dimethylcoprogen BGC (node_221_length_57612_cov_30.100447, 29,375 nt, start: 28237, end: 57612). The sequence analysis revealed the presence of eight open reading frames, $g 7985-g 7992$, of which at least 5 genes are highly likely to be involved in dimethylcoprogen biosynthesis. The gene $g 7985$ encodes a putative transmembrane transporter, g7986 encodes for a homologue to ABC transmembrane transporter, $g 7987$ encodes for a homologue to oxidoreductase, $g 7988$ encodes for a homologue to the siderophore iron transporter, $g 7989$ encodes for a homologue to acetyltransferase activity, g7990 encodes for a homologue to long-chain acyl-CoA synthetase, $g 7991$ encodes for a NRPS protein, and $g 7992$ encodes for a hypothetical protein of unknown function. Sequence comparison using BLASTN analysis indicated that the NRPS cluster of T. atroviride IMI 206040, locus: NW_014013633, 430,88 bp, start: 76225, end: 119313, showed substantial similarity (identity at nucleotide level of $86.35 \%$, cover: $87 \%$ ) in relation to that of T. hamatum FBL 587. The NRPS cluster of T. atroviride IMI 206040 putatively consisted of eight genes. The gene locus_tag TRIATDRAFT_219095 encodes for a protein with predicted transmembrane domains; the gene locus_tag TRIATDRAFT_161084 encodes for predicted MDR-type topology of transmembrane domains and nucleotide-binding folds (TMD6-NBF)2; the gene locus_tag TRIATDRAFT_80347 encodes for a hypothetical protein; the gene locus_tag TRIATDRAFT_219088 encodes for hypothetical protein with transmembrane domains; the gene locus_tag TRIATDRAFT_273644 encodes for a putative siderophore biosynthesis protein; the gene locus_tag TRIATDRAFT_152707 encodes for a NRPS-like protein, AMP-dependent synthetase and ligase; the gene locus_tag TRIATDRAFT_39887 encodes for a NRPS related to NPS6 (siderophore) of Cochliobolus heterostrophus; the gene locus_tag TRIATDRAFT_140190 encodes for a hypothetical protein. Gene clustering is represented by the arrows superposed on the horizontal black line. Intergenic spaces are not drawn in scale.

In the genome of T. hamatum FBL 587 we also identified a hypothetical gene cluster with a NRPS siderophore synthase (ProtID g775) encoding gene orthologue to that found in other Trichoderma species for the production of the intracellular siderophore ferricrocin [42]. An analysis of the T. hamatum FBL 587 genome revealed the presence of ferricrocin synthesis related genes comprised in the cluster, namely a transcription factor (ProtID g777), LOrnithine-N5-oxygenase (ProtID g776), and an GMC oxidoreductase (ProtID g774), as shown in the Figure 4. 

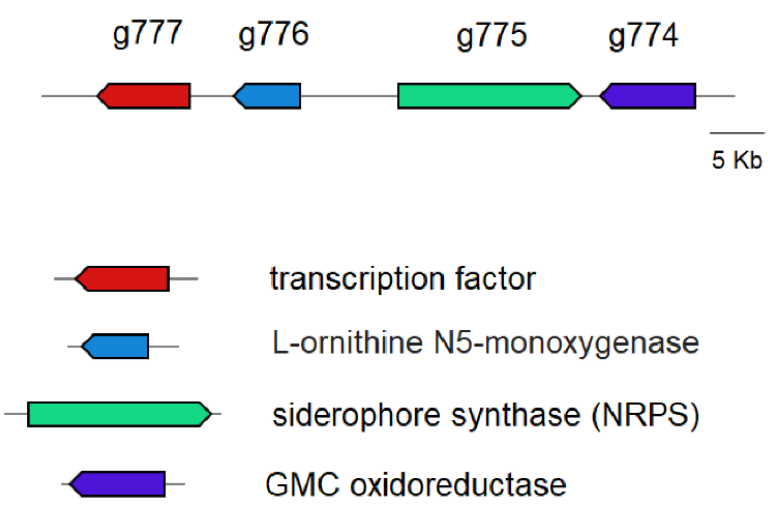

Figure 4. The putative ferricrocin siderophore gene cluster in the genome of T. hamatum FBL 587 consisted of four genes, which encode for an GMC oxidoreductase (ProtID g774), a (NRPS) ferricrocin synthase (ProtID g775), a L-Ornithine-N5-oxygenase (ProtID g776), and a transcription factor (ProtID g777). Gene clustering is represented by the arrows superposed on the horizontal black line. Intergenic spaces are not drawn in scale.

\subsection{Prediction of Genes Involved in the Metabolism of DDT}

By genomic analysis of T. hamatum FBL 587, we investigated the occurrence of genes potentially associated to DDT tolerance. Especially, protein function prediction for enzymes through GO terms allowed to specify molecular functions and potentially involvement in metabolism of aromatic compounds such as DDT. In the FBL 587 genome, there were candidate genes that might contribute to the upstream metabolism of aromatic compounds including ligninolytic enzymes such as a multicopper oxidase or extracellular laccase (ProtID g9164). We also identified genes encoding intracellular non-ligninolytic enzymes that can potentially mediate the initial (upstream) oxidation of aromatic ring structures. The degradation pathway of DDT may involve genes encoding metabolizing enzymes such as cytochrome P450 monooxygenases (CYP450s), of which the presence was revealed from the FBL 587 genome. The complete cytochrome P450 complement (CYPome) involved in resistance to polyaromatic hydrocarbons and xenobiotic detoxification (the so-called phase I enzymes; [9]) has been established from sequenced species of Trichoderma [43]. Our genomic analysis demonstrated the presence of several genes encoding cytochrome P450 monooxygenases, such as ProtID g10800, g39, g9970, g6724, g1439, g7450, g128, g8100, g9579, g3317, and g3944. In particular, T. hamatum FBL 587 has three genes encoding monooxygenases that contain domains indicating their specificity to benzoate (ProtID g39, g7471, and g9970), and one monooxygenase has specificity to n-alkane (ProtID g4242).

Additional genes that are potentially involved in the downstream steps for DDT transformation were also investigated. We identified putative genes encoding epoxide hydrolases (e.g., ProtID g352, g1543), some of which might have functions in detoxification [44]. Candidate genes encoding oxidoreductase enzymes including alcohol dehydrogenases (e.g., ProtID g7969), aldehyde dehydrogenases (e.g., ProtID g5709, g6345 and g8931), NAD-dependent aldehyde dehydrogenases (ProtID g5178, g6146, and g773), aldehyde dehydrogenases NAD (ProtID g8129, and g6040), one short-chain dehydrogenase (ProtID g1254), and FAD-dependent monooxygenases (e.g., ProtID g33, g9198, g4149, g10870, g5490, g985, g5871, and g9407) were found in the genome of FBL 587. Genes encoding dioxygenases comprising one putative hydroxyquinol-dioxygenase (ProtID g10302), and one aromatic compound dioxygenase (ProtID g10767) were also found in the T. hamatum FBL 587 genome.

Additionally, degradation of compounds such as DDT or PAHs relies on transferases (the so-called phase II conjugating enzymes; [9]) that make such polyaromatic compounds more hydrosoluble and less toxic. Our results confirmed that the T. hamatum FBL 587 
genome possesses genes encoding for glutathione S-transferases (GSTs), glycosyltransferases (GTFs), and sulfotransferases, which can catalyse the addition of glutathione, glycosyl, or sulphate donors to DDT, respectively. Specifically, 6 genes encoding putative GSTs (ProtID g920, g5585, g1796, g8350, g9635, and g8655), one GTF (ProtID g3303) and one sulfotransferase (ProtID g2564) were encoded for the potential conjugation of DDT.

According to GO functional analysis, the genes encoding transmembrane transporters (the so-called phase III coding for transporters; [9]) were also identified. These included several MFS general substrate transporters (e.g., ProtID g1350, g3396, g10401, g3264, g8078, g3803, g10322, g9334, g10400, g2310, g1362, g4159, g4828, g1309, g9714, g3742, g700, g1256, g2260, g5719, g845, g3533, and g8876), and ABC transporters (e.g., ProtID g6498, g6886, g7790, g8027, and g3411).

Finally, candidate genes encoding antioxidant enzymes, such as GSTs (see above), superoxide dismutase (SOD; ProtID g4389, and g6464) and catalase (ProtID g5890) were also found.

\subsection{Genome-Wide Transcriptomic Responses of T. hamatum FBL 587 during DDT Exposure}

In order to investigate which of the genes in T. hamatum FBL 587 were upregulated during DDT exposure in FBL 587, a genome-wide transcriptomic analysis was performed. Specifically, T. hamatum FBL 587 grown for $48 \mathrm{~h}$ with DDT $(10 \mathrm{mg} / \mathrm{L})$ was subjected to RNA-Seq analysis. For each library of RNA-Seq data, over 21-24 million reads were mapped back in pairs for both biological replicates of treated and untreated samples (Supplementary Table S1). After the alignment of the reads against the FBL 587 genome, PCA of the normalized gene expression values as input clearly separated DDT-added group (3 samples) from the control group (Figure 5). The PCA clustered DDT samples together, which formed an independent group distinct from the untreated samples (control), indicating that the gene expression profile of the DDT treatment was different from the control.

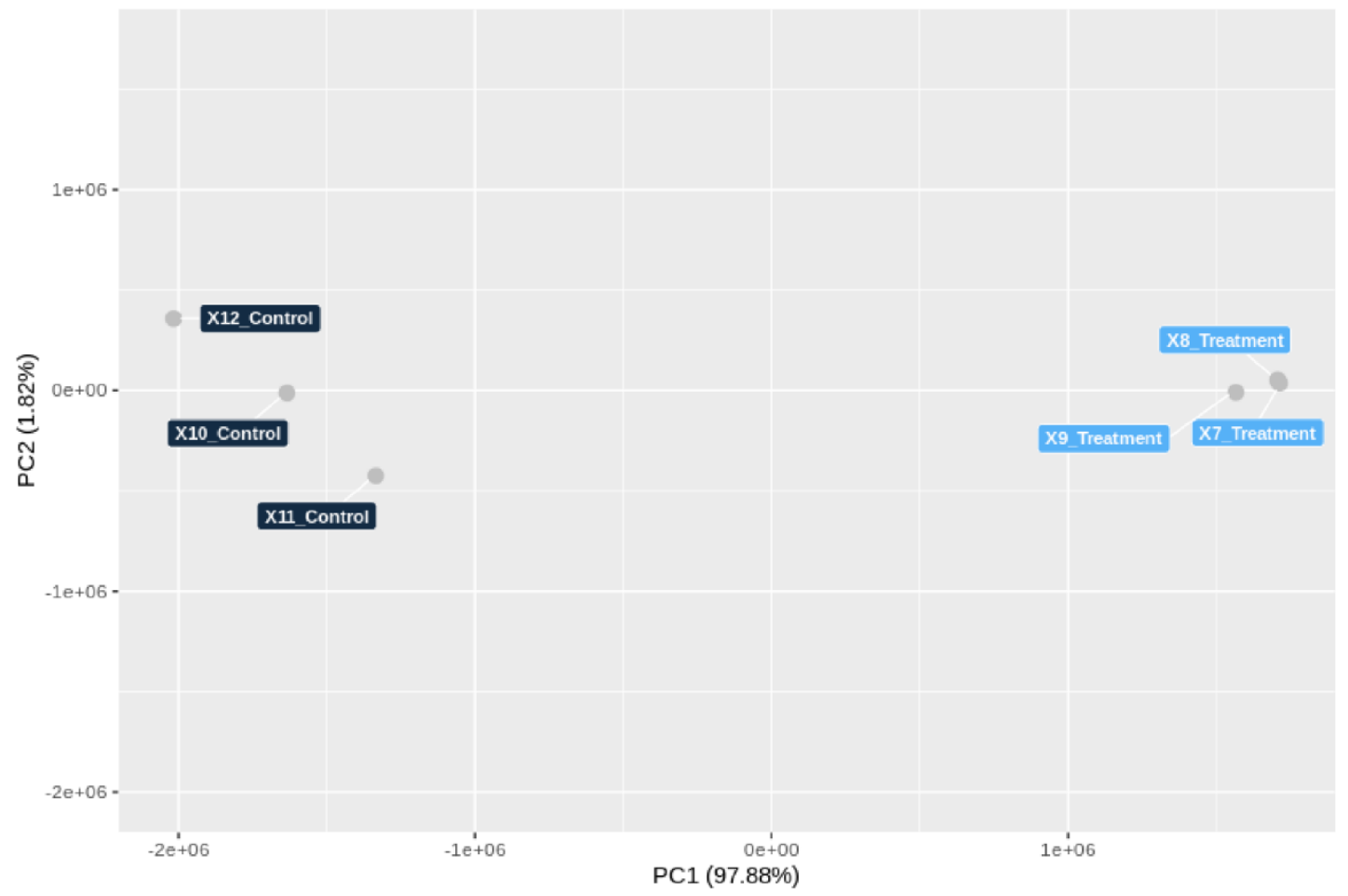

Figure 5. Principal Component Analysis (PCA) conducted on the normalized gene expression values of the samples (control and DDT treatment). X- and Y-axes show the PC1 and PC2, respectively, with the amount of variance explained by each component reported in parenthesis. Each point in the plot represents a sample, dots of the same colours are replicates of a same experimental group. PCA showed that replicates cluster together for both control and treatment, and the treatment group is clearly distinct from the control group. 
Of the 10,994 genes predicted in the FBL 587 genome, the RNA-Seq read counts to each annotated locus identified 4710 genes whose expression was regulated by DDT (FDR value $\leq 0.05$ ), as they are reported in Figure 6 . Among the significantly differentially expressed genes following exposure to DDT (FDR $\leq 0.05$ ), those with $\log 2 \mathrm{FC}$ (fold change relative to control) $\geq 1$ were upregulated genes (1706) and those with $\log 2 \mathrm{FC} \leq-1$ were downregulated genes (1770). However, the molecular function of some genes regulated by DDT could not be predicted or assigned to GO classes.

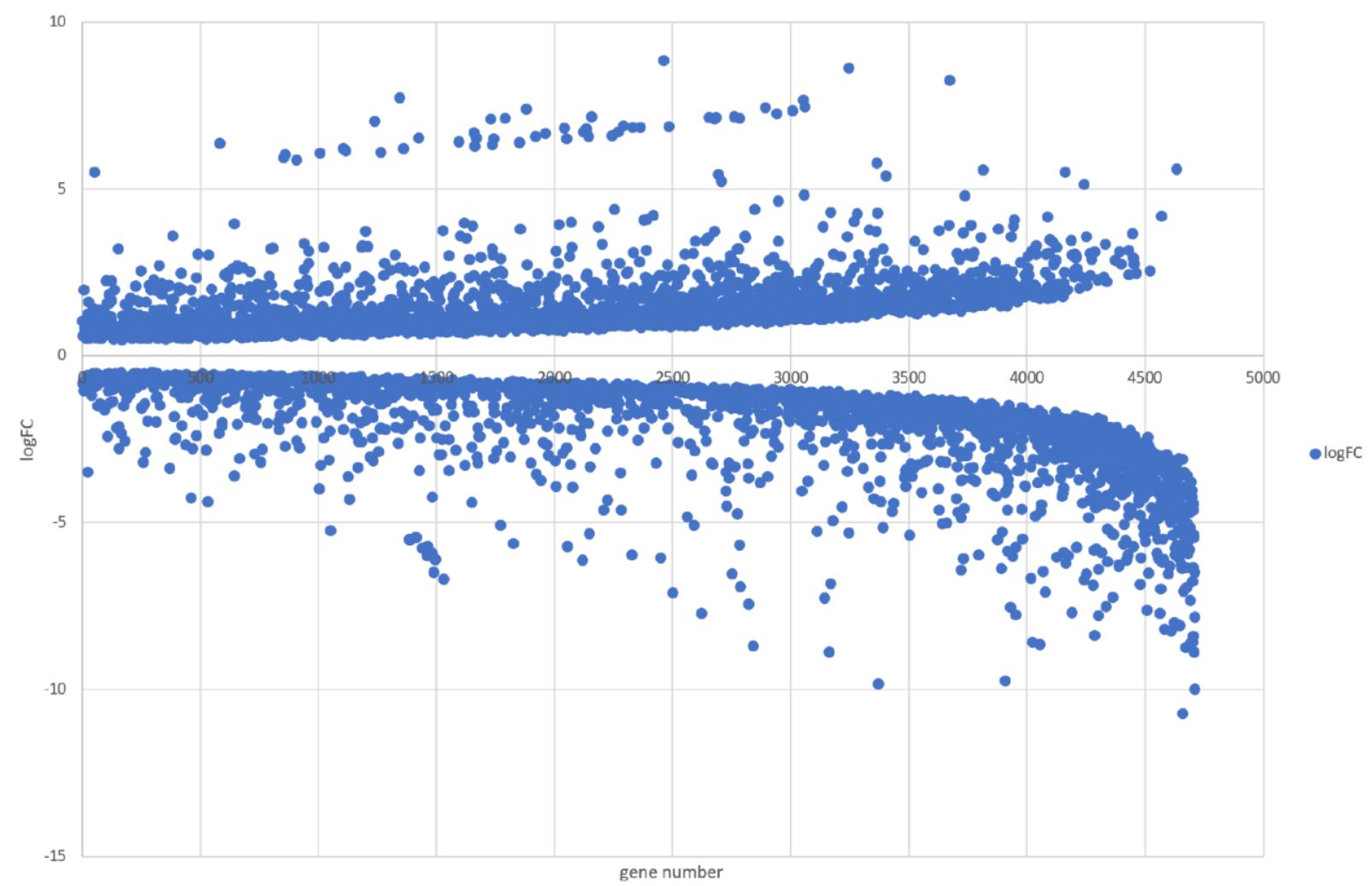

Figure 6. A plot reporting results from transcriptomic analysis for T. hamatum FBL 587 following exposure to DDT (10 mg/L for $48 \mathrm{~h}$ ), in which blue dots represent the genes (4710) that were significantly expressed (FDR $\leq 0.05$ ) by exposure to DDT, with $\log 2 \mathrm{FC} \geq 1$ for upregulated genes (1706) and $\log 2 \mathrm{FC} \leq-1$ for downregulated genes (1770), where $\mathrm{x}$-axis is the gene number and y-axis is log2FC value. Abbreviations: FDR, False Discovery Rate (Benjamini-Hochberg correction); log2FC, fold change relative to control.

\subsection{Transcriptomic Analysis of DDT Tolerance Genes by T. hamatum FBL 587}

In T. hamatum FBL 587, xenobiotic detoxification genes i.e., phase I coding for cytochrome P450 monooxygenases (CYP450s), phase II conjugating enzymes, and phase III coding for ABC efflux and MFS transporters [9] were transcriptionally upregulated $(\mathrm{FDR} \leq 0.05, \log 2 \mathrm{FC} \geq 1)$, resulting in its resistance to DDT.

With regard to phase I, the CYP450 genes ProtID g128 (cytochrome P450 monooxygenase sdnT) and ProtID g8100 (cytochrome P450 monooxygenase), which showed high expression $(\log 2 \mathrm{FC} \geq 1)$ when $T$. hamatum FBL 587 was cultured on medium with DDT, would contribute to T. hamatum tolerance to DDT.

Among the phase II conjugating enzymes, the genes encoding transferases such as GTFs (ProtID g3303) and GSTs (ProtID g1796 and g8655), which make polyaromatic compounds such as DDT or PAHs more hydrosoluble and less toxic, were significantly induced (FDR $\leq 0.05, \log 2 \mathrm{FC} \geq 1$ ) following exposure to DDT.

With regards to phase III, our genomic and transcriptomic analysis showed that in the comparison DDT treatment vs control, the genes encoding transmembrane transporters were significantly differentially expressed. Particularly, upregulation by DDT was evi- 
denced (FDR $\leq 0.05, \log 2 \mathrm{FC} \geq 1$ ) in 23 MFS transporters (ProtID g1350, g3396, g10401, g3264, g8078, g3803, g10322, g9334, g10400, g2310, g1362, g4159, g4828, g1309, g9714, g3742, g700, g1256, g2260, g5719, g845, g3533, and g8876), suggesting an important role of these transporters for cellular response to DDT. According to GO functional enrichment analysis, we also identified ABC transporters that were upregulated (e.g., ProtID g8027; FDR $\leq 0.05, \log 2 \mathrm{FC}>1$ ) during DDT exposure, suggesting their putative involvement in the translocation of DDT.

The DDT-specific upregulation of genes potentially involved in enzymatic antioxidant defence mechanisms such as superoxide dismutase (SOD, ProtID g6464; FDR $\leq 0.05$, $\log 2 \mathrm{FC}>1$ ) and catalase (ProtID g5890; FDR $\leq 0.05$, $\log 2 \mathrm{FC}>1$ ) was also observed, which in T. hamatum FBL 587 can be involved in the resistance to DDT.

\subsection{Transcriptomic Analysis of DDT Transforming Genes by T. hamatum FBL 587}

In addition to the genes involved in the detoxification of DDT by transport and metabolism (phase I, II, III; [9]), the DDT-upregulated 1706 genes included putative DDTtransforming genes. We investigated the expression profiles of enzymes for initial aromaticring oxidation, i.e., extracellular laccase and intracellular P450s. In DDT-treated samples of T. hamatum FBL 587, laccase (ProtID 9164) was not upregulated (FDR value > 0.05). However, for this gene the treatment replicates showed a higher level of expressed transcripts than control replicates. With regards to intracellular P450s, some of them were upregulated, as mentioned above.

In T. hamatum FBL 587, DDT-responsive genes potentially responsible for the downstream steps for DDT transformation were also identified. The expression patterns of these genes showed that most of them were upregulated during DDT exposure. The genes encoding epoxide hydrolases have potential functions in detoxification of xenobiotics, reducing the toxic effects of these molecules [44]). Particularly, in T. hamatum FBL 587 under DDT exposure the epoxide hydrolase (ProtID g352) resulted in an upregulated gene $(\mathrm{FDR} \leq 0.05, \log 2 \mathrm{FC}>1)$ in the process of detoxification. Moreover, a putative secreted hydrolase (ProtID g1645) resulted in significantly upregulation $(\log 2 \mathrm{FC}=6.5$ ).

Among the DDT-responsive genes, a Dye-decolorizing peroxidase (DyP-type peroxidase, ProtID g3541), some alcohol dehydrogenases (ProtID g7969), including zinccontaining alcohol dehydrogenase (ProtID g8841, g9598), and NADP-dependent alcohol dehydrogenase (ProtID g7677, and g1942) were significantly upregulated (FDR $\leq 0.05$, $\log 2 \mathrm{FC}>1$ ). Moreover, aldehyde dehydrogenases (ProtID g5709, and g6345), NADdependent aldehyde dehydrogenase (ProtID g5178, g6146), aldehyde dehydrogenase NAD (ProtID g8129, g6040) were upregulated (FDR $\leq 0.05, \log 2 \mathrm{FC}>1$ ). We also observed that two FAD-dependent monooxygenases (ProtID g9407, and g9198), and two GSTs (ProtID g1796, and g8655), and one GTF (ProtID g3303) were upregulated (FDR $\leq 0.05, \log 2 \mathrm{FC} \geq 1$ ). Interestingly, the $g 22$ gene encoding salicylate hydroxylase, which catalyses essential reactions at the junction between the so-called upper and lower catabolic pathways, presented high transcript levels (FDR $\leq 0.05, \log 2 \mathrm{FC}>1$ ) when T. hamatum FBL 587 was exposed to DDT, indicating its importance in the response to DDT.

Finally, via the genomic analysis of T. hamatum FBL 587, the genetic potential of T. hamatum FBL 587 to produce biosurfactants was investigated. Among these genes, our NGS RNA-Seq data indicated that the genes encoding hydrophobins cerato-platanins (ProtID g2774, g5696, and g150) and cerato-ulmins (ProtID 2606) were not upregulated during the two days of DDT exposure. However, the 1706 DDT-upregulated genes included genes involved in lipid transport and metabolism, in which lipases (ProtID g8227 and g4462) were upregulated (FDR $\leq 0.05, \log 2 \mathrm{FC}>1$ ). Trichoderma hamatum FBL 587 contains key genes for trehalose synthesis with the potential to extracellular trehalose lipid biosurfactants, namely trehalose-phosphate synthase (ProtID g2142), and trehalose-phosphate phosphatase (ProtID g9403). Although the trehalose-phosphate synthase was not upregulated, it resulted a differentially expressed gene $(\log 2 \mathrm{FC}=0.7)$ during the two days of exposure to DDT. 


\section{Discussion}

\subsection{Genome Properties of T. hamatum FBL 587}

Trichoderma hamatum FBL 587 showed a remarkable DDT-resistance, as reported in Russo et al., [5]. Moreover, results from this study suggest a putative ability of T. hamatum FBL 587 to enhance DDT degradation process in soil. Here we present the $38.9 \mathrm{Mb}$ genome sequence of T. hamatum FBL 587, 98.5\% complete as predicted by BUSCO, and transcriptomic analysis to observe differential expression of genes by DDT. For the first time, in T. hamatum FBL 587 we provide molecular evidence showing the DDT induced over-expression of several genes correlated with tolerance to DDT and putatively with DDT metabolism.

The genome size of T. hamatum FBL 587 is essentially similar to that of other sequenced T. hamatum strains, namely YYH13 (38.93 Mb; [13]), YYH16 (38.92 Mb; [13]), and GD12 $(38.43 \mathrm{Mb} ;[14,15])$. Moreover, the annotated 10,994 protein-coding genes in FBL 587 fell within the known range for the genomes of the T. hamatum strains YYH13 (11,316), YYH16 $(11,755)$ and GD12 $(11,203)$, as reported in [13]. The $1.2 \%$ repetitive elements found in the genome of T. hamatum FBL 587 was typical among the sequenced T. hamatum strains, i.e., $1.47 \%$ repetitive elements for $\mathrm{YYH} 13,1.58 \%$ for $\mathrm{YYH} 16$, and 1.31\% for GD12 [13]. Therefore, these NGS data indicate similar genome properties, reflecting a recent common ancestor.

This finding is also supported by further genomic analysis. In T. hamatum FBL 587 using the dbCAN CAZyme database we revealed the presence of a range of genes encoding for CAZymes that can be comparable with that of previously sequenced T. hamatum strains YYH13, YYH16 and GD12 [13-15], reflecting the adaption to their ecological niches as saprotrophic Trichoderma species in soil and rhizosphere, competing with other soil microorganisms. With respect to the GH class of CAZymes, the number of members of the glycoside hydrolases found in FBL 587 was similar to that harboured by other sequenced strains (YYH13, YYH16, and GD12) of T. hamatum. For instance, the cellulolytic CAZyme composition in T. hamatum strains YYH13, YYH16 and GD12 [13-15] was represented by the presence of frequent chitinases (GH18). Consistently with the findings, the genome of T. hamatum FBL 587 revealed 28 copies of GH18 and 3 copies of GH20. Moreover, among the carbohydrate hydrolases, the number of cellulases (e.g., GH6 = 1, GH7 = 2), glucanases $(\mathrm{GH} 55=8, \mathrm{GH} 12=3, \mathrm{GH} 17=2, \mathrm{GH} 81=2$ and GH64 = 3), xylanases (e.g., GH10 = 3, GH11 = 4), $\beta$-glucosidases and $\beta$-galactosidases $(\mathrm{GH} 1=3), \alpha$-glucosidases $(\mathrm{GH} 31=7)$, $\beta$-galactosidases $(\mathrm{GH} 35=2)$ and $\alpha$-mannanases $(\mathrm{GH76}=8)$ found in T. hamatum FBL 587 was significantly similar to that of other previously sequenced strains of T. hamatum [13].

The SM BSGC potential of T. hamatum FBL 587, which includes PKSs, NRPSs and terpenes, was compared with those of other Trichoderma species of the section Trichoderma (see [15]), such as T. atroviride IMI 2060, which is publicly available at the antiSMASH database [30], and T. lixii MUT3171 [4] of the clade Harzianum/Virens (see [15]) as outgroup. Most of the antiSMASH-6.0-detected gene clusters cannot be annotated in T. hamatum FBL 587 as their composition is not similar to any known fungal BGC. Apparently, putative BSGCs for mycotoxin production were not detected in T. hamatum FBL 587, which indicates that mycotoxin production does not play a major role in this strain, thus making the strain an ideal, harmless, candidate to avoid the risk of mycotoxin contamination during bioremediation. However, the SM responses of T. hamatum FBL 587 in the presence of DDT as well as the association between the ability to biodegrade DDT and other pollutants and mycotoxin production are to be experimentally verified, possibly under conditions closer to the level of soil DDT contamination [2], considering also factors such as light, $\mathrm{pH}$, nutrients that can be relevant in the fungal mycotoxin production [42].

Trichoderma species are reported to synthesize a broad diversity of terpenoids by terpene synthases [45], including MT $\gamma$-terpinene and the sesquiterpene (SQT) $\gamma$-candinene that were the most abundant VOCs of T. hamatum [46]. In T. hamatum FBL 587, terpenes have not been experimentally characterized yet, but in its genome we have identified terpene synthase genes (e.g., $g 7460, g 9669$, and $g 3784$ ) potentially involved in terpene biosynthesis [47]. Most of them apparently were not upregulated after two days of exposure to 
DDT. However, in T. hamatum FBL 587 a BGC putatively involved in the biosynthesis of squalestatin S1 or squalestatin analogues [37], homologue to the BGC found in other Trichoderma species including T. atroviride IMI 2060 (antiSMASH database; [29,30]), contained a squalene synthase (ProtID g4482) that was upregulated under two days of exposure to DDT, but a deeper analysis is needed to draw informed conclusions. Moreover, our antiSMASHbased analysis found a putative T1PKS gene cluster for alternariol biosynthesis in the genome of T. hamatum FBL 587. Alternariol, a compound with antioxidant activity [36], was obtained from the fermentation broth of Trichoderma sp. Jing-8 [48,49]. However, our RNASeq data showed that the T1PKS gene ( $g 8761)$ belonging to this putative gene cluster was not upregulated by DDT exposure, which indicates alternariol biosynthesis is not involved in oxidative stress tolerance. Moreover, we did not observe upregulation by DDT exposure in T. hamatum FBL 587 for the gene $g 1187$ encoding a terpene cyclase/mutase belonging to the putative biosynthetic gene cluster for clavaric acid. A detailed comparative analysis including other Trichoderma species to demonstrate genomic organization of terpene BGCs is worthy of further investigations, but it goes beyond the scope of the present paper.

Interestingly, a NRPS gene (g7991) cluster in T. hamatum FBL 587 showed an identical composition to the known BGC for the extracellular siderophore dimethylcoprogen $[40,41]$ and was upregulated in the presence of DDT. Another NRPS gene $(g 775)$ homologue of the NRPS gene responsible for the synthesis of ferricrocin [50] was identified in T. hamatum FBL 587 genome, which was arranged in a distinct cluster. However, even though it is known that the siderophore ferricrocin is used for intracellular storage of iron and that it is involved in the protection of fungal cells from oxidative stress [51], the $g 775$ was not upregulated by exposure to DDT. Therefore, although the role of siderophores in fungal resistance to oxidative stress was proposed in previous studies $[4,40,41]$, their contribution to the resistance against DDT-induced ROS should be evaluated under varying DDT contamination and exposure conditions.

\subsection{Transcriptomic Analysis of DDT Tolerance Genes by T. hamatum FBL 587}

According to our analyses, T. hamatum FBL 587 under DDT exposure upregulated phase I, II, III genes encoding different enzymes potentially involved in modification and translocation of DDT. Considering the results at the transcription of the phase I genes that regards genes encoding for CYP450s, our analysis revealed that DDT induced overexpression of genes encoding for cytochrome P450 monooxygenase sdnT (ProtID g128) and cytochrome P450 monooxygenase (ProtID g8100). Cytochrome P450 monooxygenases of fungi are involved in many essential cellular processes and play diverse roles since they catalyse the conversion of hydrophobic intermediates and have been found to support the growth of fungi also in the presence of environmental pollutants [52].

With regard to phase II, our analysis also revealed genes encoding transferases in T. hamatum FBL 587. In the comparison DDT treatment vs control, the gene encoding transferases such as GTFs (ProtID g3303) and GSTs (ProtID g1796 and g8655), which make polyaromatic compounds such as DDT more hydrosoluble and less toxic, were upregulated in response to DDT. For instance, we hypothesized that in T. hamatum FBL 587, glycosyltransferases may participate to phase II reactions in the DDT modification pathway: after the initial attack by oxidoreductases, these enzymes may transfer an activated sugar residue to the hydroxyl groups of the DDT metabolites, which may be secreted.

DDT exposure induced in T. hamatum FBL 587 the transcription of a significant number of genes, with a distinct transcriptomic response explained by genes putatively involved in translocation of DDT out of cells (phase III). For instance, some ABC transporters (e.g., ProtID g8027) were upregulated in response to DDT. This observation is consistent with previous studies in Trichoderma in which $\mathrm{ABC}$ transporters contributed to resistance against toxic compounds and were involved in protection from exogenous toxicants [9]. Also, in T. hamatum FBL 587 we found the transcription of a significantly number of genes encoding the transmembrane transporters MFS, which are known for their ability to actively export 
xenobiotic and/or metabolized compounds across the cell membranes. On the basis of these results, we hypothesized putative translocation of DDT by means of MFS transporters that not only could contribute to resistance against DDT but also could enable modification of DDT.

The transcription factor that probably is responsible for induced overexpression of the phase I, II and III genes would be worthy of further investigations. We hypothesized that it might be regulated by a putative xenobiotic detoxification regulator such as one of the members of the fungus-specific Zn2Cys6 family, which have been known to be the regulator of fungal $\mathrm{ABC}$ transporters [9].

Particularly worthy of note is the fact that the comparison between cells growth under DDT exposure vs control evidenced among the upregulation of genes encoding oxidoreductases of T. hamatum FBL 587, a significantly differential expression of a gene for superoxide dismutase (ProtID g6464). It is well known that within the soil community, Trichoderma species respond to ROS originated during the plant-pathogenic fungi interactions by enhancing the expression of genes that encode for oxidative stress response and detoxification enzymes [12]. Since DDT is known to increase the production of ROS in T. hamatum FBL 587 , leading to detectable oxidative stress [5], significant upregulation of ROS scavenging enzymes in cells exposed to DDT was expected and was confirmed by the transcriptomic analysis.

\subsection{Transcriptomic Analysis of DDT Transforming Genes by T. hamatum FBL 587}

DDT biodegradation in soil was assumed to be mediated by T. hamatum FBL 587, since its inoculation favoured the biodegradation of the compound into DDT isomers and its metabolites DDE and DDD and decreased the overall DDT concentration in soil by inducing an increased accumulation of the pollutants in roots of $C$. pepo. It is worth noting that all the genes involved in the fungal DDT biodegradation pathway have not yet been elucidated. Therefore, the putative DDT metabolism by T. hamatum FBL 587 requires to be corroborated by degradation studies with DDT as sole carbon source. Moreover, the effect of autochthonous bacterial enzymes such as DDT-dehydrohalogenases that can be involved in the catalytic degradation of DDT to form DDD/DDE [53] cannot be ruled out in our DDT-contaminated soil.

Although the genetic details of fungal DDT degradation have not been established, the genomic and transcriptomic analysis of T. hamatum FBL 587 carried out in this study revealed a total of 1706 genes upregulated by DDT, many of which having known molecular functions. Several of these genes were encoding for enzyme systems potentially involved in xenobiotic degradation such as multicopper laccases and peroxidases. The presence of gene products in the RNA-Seq analysis of T. hamatum FBL 587 identified as an extracellular laccase could also suggest the putative involvement of this enzyme in the biodegradation process, even though it was not upregulated during the two days of DDT exposure. Indeed, the degradation of PAHs by laccase activity of T. asperellum was nearly undetectable during the initial two days but increased significantly after four days [7].

The initial steps of PAH metabolism in many filamentous fungi involve the action of cytochrome P450 monooxygenases and epoxide hydrolases [52]. The analyses performed in this study suggested that the ability of T. hamatum FBL 587 to metabolize DDT lies in key enzymatic reactions of aerobic biodegradation catalysed by peroxidases and cytochrome P450 oxidases. Indeed, in response to DDT, it upregulated genes for cellular oxidant detoxification, such as a catalase-peroxidase (ProtID g5890), a putative secreted hydrolase (ProtID g1645), and a DyP-type peroxidase (ProtID g3541). Such gene regulation pattern would suggest that in T. hamatum FBL 587 the cleavage of carbon-chlorine bonds in organochlorine compounds degradation may occur via enzymatic dechlorination catalysed by enzymes such as monooxygenases.

Moreover, T. hamatum FBL 587 also upregulated genes encoding intracellular enzymes such as cytochrome P450 monooxygenase (CYPs or P450s), which are key enzymes in fungal xenobiotic detoxification [54]. P450 enzymes have been reported to metabolize DDT 
under aerobic conditions as well as to be involved in the degradation of some polyaromatic pollutants such as PAHs [4]. In Trichoderma species, cytochrome P450s form an important group of enzymes involved in degradation of environmental pollutants [43]. It can be assumed that intracellular P450 enzymes also have an important role in the metabolism of persistent aromatic compounds in T. hamatum FBL 587. Its transcriptomic analysis revealed that P450s might be responsible for the initial oxidation of aromatic rings. A previous genome-scale identification of $\mathrm{P} 450$ genes in T. lixii showed potential DDT-oxidizing activity of these enzymes [4]. Understanding the molecular mechanisms of fungal P450 genes involved in DDT metabolism of T. hamatum FBL 587 is the objective of ongoing study.

Intriguingly, the gene $g 4985$ encoding benzoate 4-monooxygenase, which is responsible for cytochrome P450 dependent benzoate hydroxylation in microsomes [55], was upregulated, while three genes for putative benzoate 4-monooxygenase cytochrome P450 (ProtID g39, g7471, and g9970) were not. Moreover, DDT exposure induced upregulation and differentially expression of two genes encoding dihydroxybenzoate decarboxylase (ProtID g24, g7819). This enzyme is involved in the pathway benzoate degradation via hydroxylation, which is part of aromatic compound metabolism [56]. On the other hand, the gene $g 21$ for catechol 1,2-dioxygenase showed higher expression when T. hamatum FBL 587 was cultured on medium with DDT than control, but apparently it was not significantly upregulated during the two days of exposure to DDT. It thus appeared that the regulation of related genes could be differentially modulated by the xenobiotic stress [57].

In addition to P450s, in response to DDT we observed upregulation of many other genes encoding potential DDT-transforming enzymes such as alcohol dehydrogenase (ProtID g8841, g9598), aldehyde dehydrogenase (e.g., ProtID g8129), FAD-dependent monooxygenases (ProtID g9407 and g9198), GTF (ProtID 3303), and GST (ProtID 1796, 8655). Particularly worthy of note is the fact that at least eight aldehyde dehydrogenases were identified in the T. hamatum FBL 587 genome and five of them were upregulated in terms of mRNA levels after cell exposure to DDT. Aldehyde dehydrogenases could exhibit oxidase activities to catalyse the oxidation of DDT intermediate, such as phenoxybenzyl aldehyde to phenoxybenzoic acid [58]. Moreover, the gene $g 22$ for salicylate hydroxylase was also upregulated in response to DDT exposure, which in the microbial degradation of polycyclic aromatic hydrocarbons, catalyses essential reactions at the junction between the so-called upper and lower catabolic pathways [59].

Upregulation of T. hamatum FBL 587 genes that might increase the production of lipid biosurfactants to emulsify DDT and of genes involved in lipid transport and metabolism, among them lipases (ProtID g8227, g4462), in response to exposure to DDT could also be associated to the role that biosurfactants with bioemulsifier activities may have in biotransformation of aromatic compounds though enhancing their hydrosolubility and bioavailability into the soil [4]. Trichoderma hamatum FBL 587 resulted to have also the genetic potential to produce other biosurfactants such as hydrophobins, which genes were deduced from genomic sequences of Trichoderma species [12]. In saprophytic Trichoderma species established in soil and rhizosphere, hydrophobin-like proteins are involved in attachment of Trichoderma to roots [42]. In agreement with this finding, in the genome of T. hamatum FBL 587 genes encoding hydrophobin cerato-platanins and cerato-ulmins (small amphiphilic proteins) were found, but NGS RNA-Seq data showed that they were not upregulated during two days of DDT exposure. However, in T. hamatum FBL 587 the gene encoding trehalose-phosphate synthase (ProtID g2142), a key gene for trehalose synthesis, resulted to be transcripted, though at low level, in response to the 2-day DDT exposure stress, suggesting the involvement, to some extent, of the trehalose metabolism in increasing the tolerance to DDT. It seems reasonable to assume that the production of extracellular trehalose lipid biosurfactants with emulsifying properties would contribute to increase tolerance of T. hamatum FBL 587 to DDT and/or to the enhanced uptake of DDT from the plant root system, as observed in the pot trial. 


\section{Conclusions}

The whole-genomic and transcriptomic analysis of T. hamatum FBL 587, performed for the first time, provided insights into the complex gene responses of this strain when challenged by DDT exposure. Several T. hamatum FBL 587 genes were upregulated in association with DDT exposure, which suggest their putative roles in DDT metabolism. However, the elucidation of the exact molecular mechanisms associated with them requires further studies. In addition, upregulation of many genes with unknown molecular functions provides a clue for future research into the identification of novel genes involved in DDT biodegradation. Further studies involving RNA-Seq data are necessary to characterize the transcriptional regulation of the system of DDT detoxification genes in T. hamatum FBL 587. Understanding the genetic aspects involved in DDT metabolization could support the exploitation of T. hamatum FBL 587 for the mycoremediation of DDT-polluted environments. Future research should include molecular approaches that allow real-time comparison of the functionality of different genes inducing tolerance or degradation of DDT for the efficient screening and application of this strain in mycoremediation strategies.

Supplementary Materials: The following are available online at https: / www.mdpi.com/article/ 10.3390/microorganisms9081680/s1, Figure S1: Low expression filtering step on transcriptome sequencing data from Trichoderma hamatum FBL 587; the algorithm calculated a Global Jaccard index of similarity (on the Y-axis) between the samples in function of different minimum normalized read counts (on the X-axis). The graphic shows the threshold at $s=13.294$ in which the replicates have the highest similarity; Table S1: Number of NGS-RNASeq reads before and after quality check on the raw sequencing data from Trichoderma hamatum FBL 587; Table S2: Trimmed Mean of M-values (TMM) obtained by normalization applied to the raw fragment from Trichoderma hamatum FBL 587; Table S3: Reads Per Kilobase Million (RPKM) obtained by normalization applied to the raw fragment from Trichoderma hamatum FBL 587; Table S4: The concentrations of DDT metabolites and isomers detected in soil and plant organs $(\mathrm{mg} / \mathrm{kg})$ of two Cucurbita pepo accessions inoculated with Trichoderma hamatum FBL 587. ND—not detected, $\Sigma$ DDT = sum of DDT metabolites and isomers; Table S5: Carbohydrate active enzymes (CAZymes) in Trichoderma hamatum FBL 587 identified with the dbCAN2, which integrates three different tools: (1) HMMER, (2) DIAMOND, (3) Hotpep (see text); Table S6: The secondary metabolite gene clusters identification in Trichoderma hamatum FBL 587 performed with antiSMASH v. 6.0 .0 (see text); Table S7: The secondary metabolite gene clusters identification in Trichoderma asperellum CBS 433.97 performed with antiSMASH v. 6.0.0 (see text); Table S8: The secondary metabolite gene clusters identification in Trichoderma atroviride IMI 206040 performed with antiSMASH v. 6.0.0 (see text); Table S9: The secondary metabolite gene clusters identification in Trichoderma gamsii T6085 performed with antiSMASH v. 6.0.0 (see text); Table S10: The secondary metabolite gene clusters identification in Trichoderma hamatum GD12 performed with antiSMASH v. 6.0.0 (see text); Table S11: The secondary metabolite gene clusters identification in Trichoderma lixii MUT3171 performed with antiSMASH v. 6.0.0 (see text).

Author Contributions: Conceptualization, A.M.P. and D.D.; Conceptualization pot trials E.M., M.T.; methodology, A.M.P., D.D., F.R., L.C., E.M.F. and M.T.; validation, A.M.P. and D.D.; formal analysis, D.D.; investigation, F.R., L.C., E.M., E.M.F. and M.T.; data curation, A.M.P., D.D., E.M.F., M.T., A.C. and O.M.; writing-original draft preparation, D.D., E.M.F., M.T., L.C. and A.C.; writingreview and editing, A.M.P., D.D. and E.M.; visualization, A.C.; supervision, A.M.P. and D.D.; project administration, A.M.P.; funding acquisition, E.M., A.M.P. and D.D. All authors have read and agreed to the published version of the manuscript.

Funding: This research received no external funding.

Institutional Review Board Statement: Not applicable.

Informed Consent Statement: Not applicable.

Data Availability Statement: The whole genome sequence project of FBL 587 has been deposited at DDBJ/ENA/GenBank under the accession SEIV00000000. The RNA-sequencing data have been deposited in NCBI SRA under the IDs: SRR14056734-9. 


\begin{abstract}
Acknowledgments: The transcriptomic study was funded by the Sapienza University, Rome, Italy. We acknowledge the Department of Technological Innovations and Safety of Plants, Products and Anthropic Settlements (DIT), INAIL, Research Area, Rome, Italy, for financially supporting the genome sequencing as part of the NGS Project in the PAR 2019-2021. We would like to thank Riccardo Aiese Cigliano (Sequentia Biotech) for suggestions in bioinformatics. The financial support for the DDT degradation study by the Polish Ministry of Agriculture and Rural Development to EM is acknowledged. The technical support from Kathleen R. Reitsma, Curator of Vegetable Crops at the US National Plant Germplasm System, and the provision of seeds for the C. pepo accessions is also kindly acknowledged. We also acknowledge the valuable comments and suggestions of two anonymous reviewers.
\end{abstract}

Conflicts of Interest: The authors declare no conflict of interest.

\title{
References
}

1. Hung, H.; Katsoyiannis, A.A.; Guardans, R. Ten Years of Global Monitoring under the Stockholm Convention on Persistent Organic Pollutants (POPs): Trends, Sources and Transport Modelling. Environ. Pollut. 2016, 217, 1-3. [CrossRef]

2. Silva, V.; Mol, H.G.J.; Zomer, P.; Tienstra, M.; Ritsema, C.J.; Geissen, V. Pesticide Residues in European Agricultural Soils-A Hidden Reality Unfolded. Sci. Total Environ. 2019, 653, 1532-1545. [CrossRef]

3. Malusá, E.; Tartanus, M.; Danelski, W.; Miszczak, A.; Szustakowska, E.; Kicińska, J.; Furmanczyk, E.M. Monitoring of DDT in Agricultural Soils under Organic Farming in Poland and the Risk of Crop Contamination. Environ. Manag. 2020, 66, 916-929. [CrossRef]

4. Venice, F.; Davolos, D.; Spina, F.; Poli, A.; Prigione, V.P.; Varese, G.C.; Ghignone, S. Genome Sequence of Trichoderma Lixii MUT3171, a Promising Strain for Mycoremediation of PAH-Contaminated Sites. Microorganisms 2020, 8, 1258. [CrossRef]

5. Russo, F.; Ceci, A.; Pinzari, F.; Siciliano, A.; Guida, M.; Malusà, E.; Tartanus, M.; Miszczak, A.; Maggi, O.; Persiani, A.M. Bioremediation of Dichlorodiphenyltrichloroethane (DDT)-Contaminated Agricultural Soils: Potential of Two Autochthonous Saprotrophic Fungal Strains. Appl. Environ. Microbiol. 2019, 85, e01720-19. [CrossRef] [PubMed]

6. Tripathi, P.; Singh, P.C.; Mishra, A.; Chauhan, P.S.; Dwivedi, S.; Bais, R.T.; Tripathi, R.D. Trichoderma: A Potential Bioremediator for Environmental Clean Up. Clean Technol. Environ. Policy 2013, 15, 541-550. [CrossRef]

7. Zafra, G.; Moreno-Montaño, A.; Absalón, Á.E.; Cortés-Espinosa, D.V. Degradation of Polycyclic Aromatic Hydrocarbons in Soil by a Tolerant Strain of Trichoderma Asperellum. Environ. Sci. Pollut. Res. 2015, 22, 1034-1042. [CrossRef] [PubMed]

8. Zafra, G.; Cortés-Espinosa, D.V. Biodegradation of Polycyclic Aromatic Hydrocarbons by Trichoderma Species: A Mini Review. Environ. Sci. Pollut. Res. 2015, 22, 19426-19433. [CrossRef] [PubMed]

9. Sang, H.; Hulvey, J.P.; Green, R.; Xu, H.; Im, J.; Chang, T.; Jung, G. A Xenobiotic Detoxification Pathway through Transcriptional Regulation in Filamentous Fungi. mBio 2018, 9, e00457-18. [CrossRef]

10. Jeong, C.-B.; Kim, H.-S.; Kang, H.-M.; Lee, J.-S. ATP-Binding Cassette (ABC) Proteins in Aquatic Invertebrates: Evolutionary Significance and Application in Marine Ecotoxicology. Aquat. Toxicol. 2017, 185, 29-39. [CrossRef] [PubMed]

11. Olicón-Hernández, D.R.; González-López, J.; Aranda, E. Overview on the Biochemical Potential of Filamentous Fungi to Degrade Pharmaceutical Compounds. Front. Microbiol. 2017, 8, 1792. [CrossRef]

12. Druzhinina, I.S.; Seidl-Seiboth, V.; Herrera-Estrella, A.; Horwitz, B.A.; Kenerley, C.M.; Monte, E.; Mukherjee, P.K.; Zeilinger, S.; Grigoriev, I.V.; Kubicek, C.P. Trichoderma: The Genomics of Opportunistic Success. Nat. Rev. Microbiol. 2011, 9, 749-759. [CrossRef] [PubMed]

13. Cheng, P.; Liu, B.; Su, Y.; Hu, Y.; Hong, Y.; Yi, X.; Chen, L.; Su, S.; Chu, J.S.C.; Chen, N.; et al. Genomics Insights into Different Cellobiose Hydrolysis Activities in Two Trichoderma Hamatum Strains. Microb. Cell Fact. 2017, 16, 1-16. [CrossRef] [PubMed]

14. Studholme, D.J.; Harris, B.; Le Cocq, K.; Winsbury, R.; Perera, V.; Ryder, L.; Ward, J.L.; Beale, M.H.; Thornton, C.R.; Grant, M. Investigating the Beneficial Traits of Trichoderma Hamatum GD12 for Sustainable Agriculture-Insights from Genomics. Front. Plant Sci. 2013, 4. [CrossRef]

15. Kubicek, C.P.; Steindorff, A.S.; Chenthamara, K.; Manganiello, G.; Henrissat, B.; Zhang, J.; Cai, F.; Kopchinskiy, A.G.; Kubicek, E.M.; Kuo, A.; et al. Evolution and Comparative Genomics of the Most Common Trichoderma Species. BMC Genom. 2019, $20,1-24$. [CrossRef] [PubMed]

16. White, J.C.; Zeeb, B.A. Plant Phylogeny and the Remediation of Persistent Organic Pollutants. In Phytoremediation; Willey, N., Ed.; Methods in Biotechnology; Humana Press: Totowa, NJ, USA, 2007; Volume 23, pp. 71-87, ISBN 978-1-58829-541-5.

17. Andrews, S. FastQC: A Quality Control Tool for High Throughput Sequence Data. 2010. Available online: http://www. Bioinformatics.Babraham.Ac.Uk/Projects (accessed on 24 January 2020).

18. Bolger, A.M.; Lohse, M.; Usadel, B. Trimmomatic: A Flexible Trimmer for Illumina Sequence Data. Bioinformatics 2014, 30, 2114-2120. [CrossRef]

19. Bankevich, A.; Nurk, S.; Antipov, D.; Gurevich, A.A.; Dvorkin, M.; Kulikov, A.S.; Lesin, V.M.; Nikolenko, S.I.; Pham, S.; Prjibelski, A.D.; et al. SPAdes: A New Genome Assembly Algorithm and Its Applications to Single-Cell Sequencing. J. Comput. Biol. 2012, 19, 455-477. [CrossRef] 
20. Nikolenko, S.I.; Korobeynikov, A.I.; Alekseyev, M.A. BayesHammer: Bayesian Clustering for Error Correction in Single-Cell Sequencing. BMC Genom. 2013, 14, S7. [CrossRef]

21. Gurevich, A.; Saveliev, V.; Vyahhi, N.; Tesler, G. QUAST: Quality Assessment Tool for Genome Assemblies. Bioinformatics 2013, 29, 1072-1075. [CrossRef]

22. Lagesen, K.; Hallin, P.; Rødland, E.A.; Stærfeldt, H.-H.; Rognes, T.; Ussery, D.W. RNAmmer: Consistent and Rapid Annotation of Ribosomal RNA Genes. Nucleic Acids Res. 2007, 35, 3100-3108. [CrossRef] [PubMed]

23. Lowe, T.M.; Chan, P.P. TRNAscan-SE On-Line: Integrating Search and Context for Analysis of Transfer RNA Genes. Nucleic Acids Res. 2016, 44, W54-W57. [CrossRef] [PubMed]

24. Smit, A.F.A.; Hubley, R.; Green, P. RepeatMasker Open-4.0. 2018. Available online: http:/ / Repeatmasker.Org/ (accessed on 1 July 2020).

25. Manchanda, N.; Portwood, J.L.; Woodhouse, M.R.; Seetharam, A.S.; Lawrence-Dill, C.J.; Andorf, C.M.; Hufford, M.B. GenomeQC: A Quality Assessment Tool for Genome Assemblies and Gene Structure Annotations. BMC Genom. 2020, 21, 193. [CrossRef] [PubMed]

26. Hoff, K.J.; Stanke, M. Predicting Genes in Single Genomes with AUGUSTUS. Curr. Protoc. Bioinform. 2019, 65, e57. [CrossRef] [PubMed]

27. Törönen, P.; Medlar, A.; Holm, L. PANNZER2: A Rapid Functional Annotation Web Server. Nucleic Acids Res. 2018, 46, W84-W88. [CrossRef]

28. Zhang, H.; Yohe, T.; Huang, L.; Entwistle, S.; Wu, P.; Yang, Z.; Busk, P.K.; Xu, Y.; Yin, Y. DbCAN2: A Meta Server for Automated Carbohydrate-Active Enzyme Annotation. Nucleic Acids Res. 2018, 46, W95-W101. [CrossRef]

29. Blin, K.; Shaw, S.; Kloosterman, A.M.; Charlop-Powers, Z.; van Wezel, G.P.; Medema, M.H.; Weber, T. AntiSMASH 6.0: Improving Cluster Detection and Comparison Capabilities. Nucleic Acids Res. 2021, gkab335. [CrossRef]

30. Blin, K.; Shaw, S.; Kautsar, S.A.; Medema, M.H.; Weber, T. The AntiSMASH Database Version 3: Increased Taxonomic Coverage and New Query Features for Modular Enzymes. Nucleic Acids Res. 2021, 49, D639-D643. [CrossRef]

31. Dobin, A.; Davis, C.A.; Schlesinger, F.; Drenkow, J.; Zaleski, C.; Jha, S.; Batut, P.; Chaisson, M.; Gingeras, T.R. STAR: Ultrafast Universal RNA-Seq Aligner. Bioinformatics 2013, 29, 15-21. [CrossRef]

32. Liao, Y.; Smyth, G.K.; Shi, W. FeatureCounts: An Efficient General Purpose Program for Assigning Sequence Reads to Genomic Features. Bioinformatics 2014, 30, 923-930. [CrossRef]

33. Rau, A.; Gallopin, M.; Celeux, G.; Jaffrézic, F. Data-Based Filtering for Replicated High-Throughput Transcriptome Sequencing Experiments. Bioinformatics 2013, 29, 2146-2152. [CrossRef] [PubMed]

34. Baroncelli, R.; Zapparata, A.; Piaggeschi, G.; Sarrocco, S.; Vannacci, G. Draft Whole-Genome Sequence of Trichoderma Gamsii T6085, a Promising Biocontrol Agent of Fusarium Head Blight on Wheat. Genome Announc. 2016, 4, e01747-15. [CrossRef]

35. Shi-Kunne, X.; Seidl, M.F.; Faino, L.; Thomma, B.P.H.J. Draft Genome Sequence of a Strain of Cosmopolitan Fungus Trichoderma Atroviride. Genome Announc. 2015, 3, e00287-15. [CrossRef]

36. Chooi, Y.-H.; Muria-Gonzalez, M.J.; Mead, O.L.; Solomon, P.S. SnPKS19 Encodes the Polyketide Synthase for Alternariol Mycotoxin Biosynthesis in the Wheat Pathogen Parastagonospora Nodorum. Appl. Environ. Microbiol. 2015, 81, 5309-5317. [CrossRef] [PubMed]

37. Bonsch, B.; Belt, V.; Bartel, C.; Duensing, N.; Koziol, M.; Lazarus, C.M.; Bailey, A.M.; Simpson, T.J.; Cox, R.J. Identification of Genes Encoding Squalestatin S1 Biosynthesis and in Vitro Production of New Squalestatin Analogues. Chem. Commun. 2016, 52, 6777-6780. [CrossRef]

38. Godio, R.P.; Fouces, R.; Martín, J.F. A Squalene Epoxidase Is Involved in Biosynthesis of Both the Antitumor Compound Clavaric Acid and Sterols in the Basidiomycete H. Sublateritium. Chem. Biol. 2007, 14, 1334-1346. [CrossRef]

39. Godio, R.P.; Martín, J.F. Modified Oxidosqualene Cyclases in the Formation of Bioactive Secondary Metabolites: Biosynthesis of the Antitumor Clavaric Acid. Fungal Genet. Biol. 2009, 46, 232-242. [CrossRef]

40. Chen, L.-H.; Lin, C.-H.; Chung, K.-R. A Nonribosomal Peptide Synthetase Mediates Siderophore Production and Virulence in the Citrus Fungal Pathogen Alternaria Alternata: Nonribosomal Peptide Synthetase of Alternaria. Mol. Plant Pathol. 2013, 14, 497-505. [CrossRef]

41. Chen, L.-H.; Yang, S.L.; Chung, K.-R. Resistance to Oxidative Stress via Regulating Siderophore-Mediated Iron Acquisition by the Citrus Fungal Pathogen Alternaria Alternata. Microbiology 2014, 160, 970-979. [CrossRef] [PubMed]

42. Mukherjee, P.K.; Horwitz, B.A.; Herrera-Estrella, A.; Schmoll, M.; Kenerley, C.M. Trichoderma Research in the Genome Era. Annu. Rev. Phytopathol. 2013, 51, 105-129. [CrossRef] [PubMed]

43. Chadha, S.; Mehetre, S.T.; Bansal, R.; Kuo, A.; Aerts, A.; Grigoriev, I.V.; Druzhinina, I.S.; Mukherjee, P.K. Genome-Wide Analysis of Cytochrome P450s of Trichoderma Spp.: Annotation and Evolutionary Relationships. Fungal Biol. Biotechnol. 2018, 5, 1-15. [CrossRef] [PubMed]

44. de Oliveira, G.S.; Adriani, P.P.; Ribeiro, J.A.; Morisseau, C.; Hammock, B.D.; Dias, M.V.B.; Chambergo, F.S. The Molecular Structure of an Epoxide Hydrolase from Trichoderma Reesei in Complex with Urea or Amide-Based Inhibitors. Int. J. Biol. Macromol. 2019, 129, 653-658. [CrossRef] [PubMed]

45. Vicente, I.; Baroncelli, R.; Morán-Diez, M.E.; Bernardi, R.; Puntoni, G.; Hermosa, R.; Monte, E.; Vannacci, G.; Sarrocco, S. Combined Comparative Genomics and Gene Expression Analyses Provide Insights into the Terpene Synthases Inventory in Trichoderma. Microorganisms 2020, 8, 1603. [CrossRef] [PubMed] 
46. Guo, Y.; Ghirardo, A.; Weber, B.; Schnitzler, J.-P.; Benz, J.P.; Rosenkranz, M. Trichoderma Species Differ in Their Volatile Profiles and in Antagonism toward Ectomycorrhiza Laccaria Bicolor. Front. Microbiol. 2019, 10, 891. [CrossRef] [PubMed]

47. Bian, G.; Han, Y.; Hou, A.; Yuan, Y.; Liu, X.; Deng, Z.; Liu, T. Releasing the Potential Power of Terpene Synthases by a Robust Precursor Supply Platform. Metab. Eng. 2017, 42, 1-8. [CrossRef] [PubMed]

48. Zhang, J.; Chen, G.-Y.; Li, X.-Z.; Hu, M.; Wang, B.-Y.; Ruan, B.-H.; Zhou, H.; Zhao, L.-X.; Zhou, J.; Ding, Z.-T.; et al. Phytotoxic, Antibacterial, and Antioxidant Activities of Mycotoxins and Other Metabolites from Trichoderma sp. Nat. Prod. Res. 2017, 31, 2745-2752. [CrossRef] [PubMed]

49. Li, M.-F.; Li, G.-H.; Zhang, K.-Q. Non-Volatile Metabolites from Trichoderma spp. Metabolites 2019, 9, 58. [CrossRef]

50. Fanelli, F.; Liuzzi, V.C.; Logrieco, A.F.; Altomare, C. Genomic Characterization of Trichoderma Atrobrunneum (T. Harzianum Species Complex) ITEM 908: Insight into the Genetic Endowment of a Multi-Target Biocontrol Strain. BMC Genom. 2018, 19, 1-18. [CrossRef]

51. Eisendle, M.; Schrettl, M.; Kragl, C.; Müller, D.; Illmer, P.; Haas, H. The Intracellular Siderophore Ferricrocin Is Involved in Iron Storage, Oxidative-Stress Resistance, Germination, and Sexual Development in Aspergillus Nidulans. Eukaryot. Cell 2006, 5, 1596-1603. [CrossRef]

52. Črešnar, B.; Petrič, Š. Cytochrome P450 Enzymes in the Fungal Kingdom. Biochim. Biophys. Acta Proteins Proteom. 2011, 1814, 29-35. [CrossRef] [PubMed]

53. Peng, R.-H.; Xiong, A.-S.; Xue, Y.; Fu, X.-Y.; Gao, F.; Zhao, W.; Tian, Y.-S.; Yao, Q.-H. Microbial Biodegradation of Polyaromatic Hydrocarbons. FEMS Microbiol. Rev. 2008, 32, 927-955. [CrossRef]

54. Lah, L.; Podobnik, B.; Novak, M.; Korošec, B.; Berne, S.; Vogelsang, M.; Kraševec, N.; Zupanec, N.; Stojan, J.; Bohlmann, J.; et al. The Versatility of the Fungal Cytochrome P450 Monooxygenase System Is Instrumental in Xenobiotic Detoxification: Fungal P450 Systems in Xenobiotic Detoxification. Mol. Microbiol. 2011, 81, 1374-1389. [CrossRef]

55. Faber, B.W.; van Gorcom, R.F.M.; Duine, J.A. Purification and Characterization of Benzoate-Para-Hydroxylase, a Cytochrome P450 (CYP53A1), from Aspergillus Niger. Arch. Biochem. Biophys. 2001, 394, 245-254. [CrossRef] [PubMed]

56. Wright, J.D. Fungal Degradation of Benzoic Acid and Related Compounds. World J. Microbiol. Biotechnol. 1993, 9, 9-16. [CrossRef]

57. Villalobos-Escobedo, J.M.; Herrera-Estrella, A.; Carreras-Villaseñor, N. The Interaction of Fungi with the Environment Orchestrated by RNAi. Mycologia 2016, 108, 556-571. [CrossRef] [PubMed]

58. Lumjuan, N.; Wicheer, J.; Leelapat, P.; Choochote, W.; Somboon, P. Identification and Characterisation of Aedes Aegypti Aldehyde Dehydrogenases Involved in Pyrethroid Metabolism. PLoS ONE 2014, 9, e102746. [CrossRef] [PubMed]

59. Jouanneau, Y.; Micoud, J.; Meyer, C. Purification and Characterization of a Three-Component Salicylate 1-Hydroxylase from Sphingomonas Sp. Strain CHY-1. Appl. Environ. Microbiol. 2007, 73, 7515-7521. [CrossRef] [PubMed] 